\title{
The dark side of flipped trinification
}

\section{P.V. Dong, ${ }^{a}$ D.T. Huong, ${ }^{a}$ Farinaldo S. Queiroz, ${ }^{b, c}$ José W. F. Valle ${ }^{d}$ and C.A. Vaquera-Araujo ${ }^{e, f}$}

${ }^{a}$ Institute of Physics, Vietnam Academy of Science and Technology, 10 Dao Tan, Ba Dinh, Hanoi, Vietnam

${ }^{b}$ Max-Planck-Institut für Kernphysik, Saupfercheckweg 1, 69117 Heidelberg, Germany

${ }^{c}$ International Institute of Physics, Federal University of Rio Grande do Norte, Campus Universitário, Lagoa Nova, Natal-RN 59078-970, Brazil

${ }^{d}$ AHEP Group, Instituto de Fúsica Corpuscular - C.S.I.C./Universitat de Valencia, Edificio de Institutos de Paterna, C/Catedratico José Beltran,

2 E-46980 Paterna (Valencia), Spain

e Departamento de Física, DCI, Campus León, Universidad de Guanajuato, Loma del Bosque 103, Lomas del Campestre C.P. 37150, León, Guanajuato, México

${ }^{f}$ Consejo Nacional de Ciencia y Tecnología, Av. Insurgentes Sur 1582. Colonia Crédito Constructor, Del. Benito Juárez, C.P. 03940, Ciudad de México, México

E-mail: pvdong@iop.vast.ac.vn, dthuong@iop.vast.ac.vn, queiroz@mpi-hd.mpg.de, valle@ific.uv.es, vaquera@fisica.ugto.mx

ABStRact: We propose a model which unifies the Left-Right symmetry with the $\mathrm{SU}(3)_{L}$ gauge group, called flipped trinification, and based on the $\mathrm{SU}(3)_{C} \otimes \mathrm{SU}(3)_{L} \otimes \mathrm{SU}(3)_{R} \otimes$ $\mathrm{U}(1)_{X}$ gauge group. The model inherits the interesting features of both symmetries while elegantly explaining the origin of the matter parity, $W_{P}=(-1)^{3(B-L)+2 s}$, and dark matter stability. We develop the details of the spontaneous symmetry breaking mechanism in the model, determining the relevant mass eigenstates, and showing how neutrino masses are easily generated via the seesaw mechanism. Moreover, we introduce viable dark matter candidates, encompassing a fermion, scalar and possibly vector fields, leading to a potentially novel dark matter phenomenology.

KeYwords: Cosmology of Theories beyond the SM, Discrete Symmetries, Gauge Symmetry

ARXIV EPRINT: 1710.06951 


\section{Contents}

1 Introduction 1

2 A flipped trinification setup 3

2.1 Gauge symmetry 3

2.2 Fermion sector 3

2.3 Scalar sector 4

2.4 Spontaneous symmetry breaking 5

2.4.1 Case 1: $w, w^{\prime} \gg \Lambda \gg u, u^{\prime} \quad 5$

2.4.2 Case 2: $\Lambda \gg w, w^{\prime} \gg u, u^{\prime} \quad 5$

2.4.3 Case $3: w, w^{\prime} \sim \Lambda \quad 6$

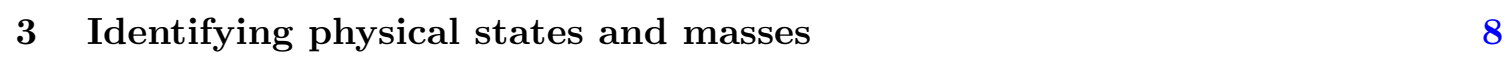

3.1 Fermion sector 8

3.2 Scalar sector $\quad 9$

$\begin{array}{lll}3.3 & \text { Gauge-boson sector } & 13\end{array}$

4 Dark matter $\quad \mathbf{1 5}$

$\begin{array}{lll}4.1 & \text { Scalar dark matter } & 16\end{array}$

$\begin{array}{lll}\text { 4.1.1 Relic density } & 16\end{array}$

$\begin{array}{lll}\text { 4.1.2 Direct detection } & 17\end{array}$

$\begin{array}{lll}4.2 & \text { Fermion dark matter } & 17\end{array}$

$\begin{array}{lll}\text { 4.2.1 Relic density } & 17\end{array}$

$\begin{array}{ll}\text { 4.2.2 Direct detection } & 17\end{array}$

$\begin{array}{lll}4.2 .3 \text { Collider } & 18\end{array}$

$\begin{array}{lll}4.3 & \text { Gauge-boson dark matter } & 19\end{array}$

$\begin{array}{ll}\text { 4.3.1 Relic density } & 19\end{array}$

$\begin{array}{llr}5 & \text { Conclusions } & 19\end{array}$

$\begin{array}{ll}\text { A Relevant scalar mass terms } & 20\end{array}$

$\begin{array}{lll}\text { B } & \text { Fermion gauge-boson interactions } & 21\end{array}$

\section{Introduction}

The mystery of Dark Matter (DM) is one of the biggest open questions in science [1-4]. Despite the fact that its existence has been ascertained at several distance scales of our universe, its nature has not yet been resolved and the Standard Model (SM) fails to account for it. The need to extend the SM goes beyond the DM problem, due to the existence of 
important open questions connected to neutrino masses, the cosmological baryon-number asymmetry, inflation and reheating. Besides, from the theoretical side, the SM fails to explain the existence of (just) three fermion families as well as the origin of the observed parity violation of the weak interaction. The purpose of this paper is to study how an extension of the SM addressing these two issues, while hosting a viable DM candidate.

The minimal left-right symmetric model based on the $\mathrm{SU}(3)_{C} \otimes \mathrm{SU}(2)_{L} \otimes \mathrm{SU}(2)_{R} \otimes$ $\mathrm{U}(1)_{B-L}$ gauge group, completed by a $Z_{2}$ symmetry that interchanges the left and right, is one of the most attractive extensions of the SM [5-10]. It gives a manifest understanding for the origin of parity violation in the weak interaction, neutrino mass generation as well as a framework for dark matter [11-15].

By the same token, models based on the $\mathrm{SU}(3)_{C} \otimes \mathrm{SU}(3)_{L} \otimes \mathrm{U}(1)_{N}$ gauge group, for short 3-3-1, offer plausible explanations for the number of generations and a hospitable scenario for neutrino mass generation as well as implementing a viable dark sector [1620, 20-24]. Hence it is theoretically well motivated to build a model where both groups are described in a unified way.

Indeed, models have been proposed in the context of the $\mathrm{SU}(3)_{C} \otimes \mathrm{SU}(3)_{L} \otimes \mathrm{SU}(3)_{R}$ gauge group [25-42]. Since they are based on a three copies of the $\mathrm{SU}(3)$ non-Abelian group, it has been coined the term trinification. The motivation for trinification lies in the unified description of both strong and electroweak interactions using the same non-Abelian gauge group, while incorporating nice features of both left-right and 3-3-1 gauge groups. Fully realistic models unifying left-right and 331 electroweak symmetries have, in fact, been recently proposed using a flipped trinification scenario with an extra $\mathrm{U}(1)_{X}$ factor $[40,41]$.

In this paper we focus on an interesting question, namely, can we build a model preserving the nice features of the left-right and 3-3-1 symmetries while naturally explaining the origin of the matter parity and dark matter? We argue that, using the gauge principle to extend the trinification framework, there is a compelling and minimal solution incorporating dark matter and realistic fermion masses. Such a flipped trinification setup is better motivated because inherits the good features of both left-right and $\mathrm{SU}(3)_{L} \otimes \mathrm{U}(1)_{N}$ symmetries and, in addition, elegantly addresses the origin of matter parity and dark matter stability in the context of 3-3-1 type models [43-55], while generating fermion masses with a minimal scalar sector. Indeed, it suffices to have one triplet $\left(\chi_{L}\right)$, one bitriplet $(\phi)$, one sextet $\left(\sigma_{R}\right)$ to generate realistic fermion masses, as opposed to earlier versions where another bitriplet was necessary $[39,40]$. In order to ensure left-right symmetry further copies of the scalar multiplets are required. Thus, a minimal version of trinification with exact left-right symmetry requires one bitriplet $(\phi)$, two sextets $\left(\sigma_{L}\right.$ and $\left.\sigma_{R}\right)$ and two triplets $\left(\chi_{L}\right.$ and $\left.\chi_{R}\right)$.

The rest of this paper is organized as follows: in section 2, we introduce the model with the gauge symmetry and particle content, focusing on the particles with unusual $B-L$ charges. We find the viable patterns of symmetry breaking and show that $W$-parity is a residual gauge symmetry which protects the dark matter stability. In section 3, we identify the physical fields and the corresponding masses. In section 4, we discuss the dark matter phenomenology. Finally, we summarize the results and conclude this work in section 5 . 


\section{$2 \quad$ A flipped trinification setup}

\subsection{Gauge symmetry}

Trinification is a theory of unified interactions based on the gauge symmetry $\mathrm{SU}(3)_{C} \otimes$ $\mathrm{SU}(3)_{L} \otimes \mathrm{SU}(3)_{R}$, the maximal subgroup of $E_{6}$ [25-27]. When multiplied by an Abelian group factor, $\mathrm{U}(1)_{X}$, we have the flipped trinification [40, 41],

$$
\mathrm{SU}(3)_{C} \otimes \mathrm{SU}(3)_{L} \otimes \mathrm{SU}(3)_{R} \otimes \mathrm{U}(1)_{X} .
$$

This symmetry can be obtained by left-right symmetrizing the 3-3-1 model in order to account for weak parity violation and close both $B-L$ and 3-3-1 algebras (cf. [56]). An alternative motivation is that it can be achieved from the minimal left-right symmetric model by enlarging the left and right weak isospin groups in order to resolve the number of fermion generations and accommodate dark matter (cf. [39]).

The electric charge operator is generally given by

$$
Q=T_{3 L}+T_{3 R}+\beta\left(T_{8 L}+T_{8 R}\right)+X,
$$

which reflects the left-right symmetry, where $T_{n L, R}(n=1,2,3, \ldots, 8)$ and $X$ are the $\mathrm{SU}(3)_{L, R}$ and $\mathrm{U}(1)_{X}$ generators, respectively. Note that $\beta$ is an arbitrary coefficient whose values dictate the electric charge of the new fermions present in the model.

As usual, the baryon minus lepton number is embedded as $Q=T_{3 L}+T_{3 R}+\frac{1}{2}(B-L)$, which implies that

$$
B-L=2\left[\beta\left(T_{8 L}+T_{8 R}\right)+X\right]
$$

is a residual gauge symmetry of $\mathrm{SU}(3)_{L} \otimes \mathrm{SU}(3)_{R} \otimes \mathrm{U}(1)_{X}$. Let us note that $B-L$ and $\mathrm{SU}(3)_{L}$ neither commute nor close algebraically. Therefore, the present framework, along the 3-3-1-1 gauge theory, constitute a class of models with a fully consistent formulation of gauged $B-L$ symmetry in 3-3-1 extensions of the Standard Model [45, 51, 55-57].

\subsection{Fermion sector}

The fermion content in this model results simply from the left-right symmetrization the left-handed fermion sector of the 3-3-1 model, so as to produce the right-handed fermion sector. The fermion sector is given as

$$
\begin{gathered}
\psi_{a L}=\left(\begin{array}{c}
\nu_{a L} \\
e_{a L} \\
N_{a L}^{q}
\end{array}\right) \sim\left(1,3,1, \frac{q-1}{3}\right), \quad \psi_{a R}=\left(\begin{array}{c}
\nu_{a R} \\
e_{a R} \\
N_{a R}^{q}
\end{array}\right) \sim\left(1,1,3, \frac{q-1}{3}\right), \\
Q_{\alpha L}=\left(\begin{array}{c}
d_{\alpha L} \\
-u_{\alpha L} \\
J_{\alpha L}^{-q-\frac{1}{3}}
\end{array}\right) \sim\left(3,3^{*}, 1,-\frac{q}{3}\right), \quad Q_{\alpha R}=\left(\begin{array}{c}
d_{\alpha R} \\
-u_{\alpha R} \\
J_{\alpha R}^{-q-\frac{1}{3}}
\end{array}\right) \sim\left(3,1,3^{*},-\frac{q}{3}\right), \\
Q_{3 L}=\left(\begin{array}{c}
u_{3 L} \\
d_{3 L} \\
J_{3 L}^{q+\frac{2}{3}}
\end{array}\right) \sim\left(3,3,1, \frac{q+1}{3}\right), \quad Q_{3 R}=\left(\begin{array}{c}
u_{3 R} \\
d_{3 R} \\
J_{3 R}^{q+\frac{2}{3}}
\end{array}\right) \sim\left(3,1,3, \frac{q+1}{3}\right),
\end{gathered}
$$

where $a=1,2,3$ and $\alpha=1,2$ are generation indices, and $q \equiv-(1+\sqrt{3} \beta) / 2$. 
The new fields $N_{a}$ and $J_{a}$ above are new leptons and quarks predicted by the model. It can be easily shown that all triangle anomalies vanish, since both $\mathrm{SU}(3)_{L}$ or $\mathrm{SU}(3)_{R}$ groups match the number of fermion generations to be that of fundamental colors, in agreement with the current observations [58]. This choice of fermion representations is the minimal for a flipped trinification [25-27].

\subsection{Scalar sector}

To break the gauge symmetry and generate the masses properly, we need introduce the scalar multiplets as follows,

$$
\begin{aligned}
\phi & =\left(\begin{array}{ccc}
\phi_{11}^{0} & \phi_{12}^{+} & \phi_{13}^{-q} \\
\phi_{21}^{-} & \phi_{22}^{0} & \phi_{23}^{-1-q} \\
\phi_{31}^{q} & \phi_{32}^{1+q} & \phi_{33}^{0}
\end{array}\right) \sim\left(1,3,3^{*}, 0\right), \\
\chi_{L} & =\left(\begin{array}{c}
\chi_{1}^{-q} \\
\chi_{2}^{-q-1} \\
\chi_{3}^{0}
\end{array}\right)_{L} \sim\left(1,3,1,-\frac{2 q+1}{3}\right), \\
\chi_{R} & =\left(\begin{array}{c}
\chi_{1}^{-q} \\
\chi_{2}^{-q-1} \\
\chi_{3}^{0}
\end{array}\right)_{R} \sim\left(1,1,3,-\frac{2 q+1}{3}\right), \\
\sigma_{L}= & \left(\begin{array}{ccc}
\sigma_{11}^{0} & \frac{\sigma_{12}^{-}}{\sqrt{2}} & \frac{\sigma_{13}^{q}}{\sqrt{2}} \\
\frac{\sigma_{12}^{-}}{\sqrt{2}} & \sigma_{22}^{--} & \frac{\sigma_{23}^{q-1}}{\sqrt{2}} \\
\frac{\sigma_{13}^{q}}{\sqrt{2}} & \frac{\sigma_{23}^{q-1}}{\sqrt{2}} & \sigma_{33}^{2 q}
\end{array}\right)_{L} \sim\left(1,6,1, \frac{2(q-1)}{3}\right), \\
\sigma_{R}= & \left(\begin{array}{ccc}
\sigma_{11}^{0} & \frac{\sigma_{12}^{-}}{\sqrt{2}} & \frac{\sigma_{13}^{q}}{\sqrt{2}} \\
\frac{\sigma_{12}^{-}}{\sqrt{2}} & \sigma_{22}^{--} & \frac{\sigma_{23}^{q-1}}{\sqrt{2}} \\
\frac{\sigma_{13}^{q}}{\sqrt{2}} & \frac{\sigma_{23}^{q-1}}{\sqrt{2}} & \sigma_{33}^{2 q}
\end{array}\right)_{R} \sim\left(1,1,6, \frac{2(q-1)}{3}\right),
\end{aligned}
$$

with the corresponding VEVs,

$$
\langle\phi\rangle=\frac{1}{\sqrt{2}}\left(\begin{array}{ccc}
u & 0 & 0 \\
0 & u^{\prime} & 0 \\
0 & 0 & w
\end{array}\right), \quad\left\langle\chi_{R}\right\rangle=\frac{1}{\sqrt{2}}\left(\begin{array}{c}
0 \\
0 \\
w^{\prime}
\end{array}\right), \quad\left\langle\sigma_{R}\right\rangle=\frac{1}{\sqrt{2}}\left(\begin{array}{ccc}
\Lambda & 0 & 0 \\
0 & 0 & 0 \\
0 & 0 & 0
\end{array}\right) .
$$

Note that the scalars transform as $\phi \rightarrow U_{L} \phi U_{R}^{\dagger}, \chi_{R} \rightarrow U_{R} \chi_{R}$, and $\sigma_{R} \rightarrow U_{R} \sigma_{R} U_{R}^{T}$ under $\mathrm{SU}(3)_{L} \otimes \mathrm{SU}(3)_{R}$. We emphasize that these three scalar multiplets are sufficient to generate all fermion masses. The scalar multiplets $\chi_{L}$ and $\sigma_{L}$ have been added to ensure the left-right symmetry, but they do not play any role in our phenomenology because the VEV of these fields are neglible hence contributing neither to gauge boson masses nor to the spontaneous symmetry breaking pattern. ${ }^{1}$ Therefore, for simplicity hereafter we ignore the VEVs of $\sigma_{L}, \chi_{L}$, keeping only the VEVs of $\sigma_{R}, \chi_{R}$, denoted omitting the subscript " $R$ ". We now discuss what types of spontaneous symmetry breaking patterns one may have in our model.

\footnotetext{
${ }^{1}$ They only contribute to the tiny neutrino masses.
} 


\subsection{Spontaneous symmetry breaking}

We now address the issue of which types of symmetry breaking patterns can be achieved within our model.

\subsubsection{Case 1: $w, w^{\prime} \gg \Lambda \gg u, u^{\prime}$}

In this scenario, we assume $w, w^{\prime} \gg \Lambda \gg u, u^{\prime}$, leading to the following breaking pattern,

$$
\begin{gathered}
\mathrm{SU}(3)_{C} \otimes \mathrm{SU}(3)_{L} \otimes \mathrm{SU}(3)_{R} \otimes \mathrm{U}(1)_{X} \\
\downarrow w, w^{\prime} \\
\mathrm{SU}(3)_{C} \otimes \mathrm{SU}(2)_{L} \otimes \mathrm{SU}(2)_{R} \otimes \mathrm{U}(1)_{B-L} \\
\downarrow \Lambda \\
\mathrm{SU}(3)_{C} \otimes \mathrm{SU}(2)_{L} \otimes \mathrm{U}(1)_{Y} \otimes W_{P} \\
\downarrow u, u^{\prime} \\
\mathrm{SU}(3)_{C} \otimes \mathrm{U}(1)_{Q} \otimes W_{P} .
\end{gathered}
$$

Notice that spontaneous symmetry breaking leaves the residual discrete gauge symmetry, $W_{P}$, conserved along with the electric and color charges. Let us now identify what symmetry is that. The VEV of $\sigma_{11}^{0}, \Lambda$, breaks $B-L$ since $[B-L]\left\langle\sigma_{11}^{0}\right\rangle=\sqrt{2} \Lambda \neq 0$, where $\sigma_{11}^{0}$ has $B-L=2$. The $\mathrm{U}(1)_{B-L}$ transformation that preserves the vacuum is $\left\langle\sigma_{11}^{0}\right\rangle \rightarrow e^{i \omega(B-L)}\left\langle\sigma_{11}^{0}\right\rangle=e^{i 2 \omega}\left\langle\sigma_{11}^{0}\right\rangle=\left\langle\sigma_{11}^{0}\right\rangle$, with $\omega$ as a transformation parameter.

Thus, we obtain $e^{i 2 \omega}=1$, or $\omega=m \pi$ for $m=0, \pm 1, \pm 2, \ldots$, and the surviving transformation is $M_{P}=e^{i m \pi(B-L)}=(-1)^{m(B-L)}$. Since the spin parity $(-1)^{2 s}$ is always conserved due to Lorentz symmetry, the residual discrete symmetry preserved after spontaneous symmetry breaking is $W_{P}=M_{P} \times(-1)^{2 s}$, which is actually a whole class of symmetries parameterized by $m$. Among such conserving transformations, we focus on the one with $m=3$,

$$
W_{P}=(-1)^{3(B-L)+2 s},
$$

which we call the matter parity. ${ }^{2}$ We stress that in our model, it emerges as a residual gauge symmetry,

$$
W_{P}=(-1)^{6\left[\beta\left(T_{8 L}+T_{8 R}\right)+X\right]+2 s},
$$

and it acts nontrivially on the fields with unusual (wrong) $B-L$ numbers. For details, see table 1. $W$-parity, $W_{P}$, is thus named following the "wrong" item as in previous studies.

\subsubsection{Case 2: $\Lambda \gg w, w^{\prime} \gg u, u^{\prime}$}

For $\Lambda \gg w, w^{\prime}$, the gauge symmetry is broken following a different path,

$$
\begin{gathered}
\mathrm{SU}(3)_{C} \otimes \mathrm{SU}(3)_{L} \otimes \mathrm{SU}(3)_{R} \otimes \mathrm{U}(1)_{X} \\
\downarrow \Lambda \\
\mathrm{SU}(3)_{C} \otimes \mathrm{SU}(3)_{L} \otimes \mathrm{SU}(2)_{R^{\prime}} \otimes \mathrm{U}(1)_{X^{\prime}} \otimes W_{P}^{\prime} \\
\downarrow w, w^{\prime} \\
\mathrm{SU}(3)_{C} \otimes \mathrm{SU}(2)_{L} \otimes \mathrm{U}(1)_{Y} \otimes W_{P} \\
\downarrow u, u^{\prime} \\
\mathrm{SU}(3)_{C} \otimes \mathrm{U}(1)_{Q} \otimes W_{P} .
\end{gathered}
$$

\footnotetext{
${ }^{2}$ We note that the matter parity present in our model coincides with $R$-parity in supersymmetry.
} 
The $\mathrm{SU}(2)_{R^{\prime}}$ symmetry is generated by $\left\{T_{6 R}, T_{7 R}, \frac{1}{2}\left(\sqrt{3} T_{8 R}-T_{3 R}\right)\right\}$, meaning that the left-right symmetry is initially broken in this case. The $\mathrm{U}(1)_{X^{\prime}}$ charge is $X^{\prime}=$ $\frac{\sqrt{3}+\beta}{4}\left(T_{8 R}+\sqrt{3} T_{3 R}\right)+X$, with $\beta=-(1+2 q) / \sqrt{3}$. The discrete symmetry $W_{P}^{\prime}$ takes a form, $W_{P}^{\prime}=(-1)^{m\left(\beta T_{8 R}+X\right)}$, which is a residual symmetry of a broken $\mathrm{U}(1)$ group, with $\mathrm{U}(\omega)=e^{i \omega 2\left(\beta T_{8 R}+X\right)}$ transformation. The second stage of the symmetry breaking is driven by $\phi_{33}^{0}, \chi_{3}^{0}$ fields. The VEV of $\chi_{3}^{0}$ breaks the symmetry $\mathrm{SU}(2)_{R^{\prime}} \otimes \mathrm{U}(1)_{X^{\prime}}$, while the VEV of $\phi_{33}^{0}$ breaks not only that symmetry but also $W_{P}^{\prime}$ and a $\mathrm{U}(1)$ group, with $\mathrm{U}\left(\omega^{\prime}\right)=e^{i \omega^{\prime} 2 \beta T_{8 L}}$ transformation, as a $\mathrm{SU}(3)_{L}$ subgroup. However, the VEV of $\phi_{33}^{0}$ leaves $W_{P}$ unbroken. Indeed, $\phi_{33}^{0}$ transforms under $\mathrm{U}(1)_{2 \beta T_{8 L}} \otimes W_{P}^{\prime}$ as,

$$
\phi_{33}^{0} \rightarrow \phi_{33}^{0 \prime}=e^{i \frac{2}{3}\left(\omega^{\prime}-m \pi\right)(1+2 q)} \phi_{33}^{0},
$$

which is invariant if $\omega^{\prime}=\pi\left(m+\frac{3 k}{1+2 q}\right)$ with $k=0, \pm 1, \pm 2 \ldots$ Choosing $k=0$, the residual symmetry coincides with $W_{P}$ after spin parity is included and taking $m=3$. Lastly, note that the hypercharge is

$$
Y=\beta T_{8 L}+\frac{\sqrt{3} \beta-1}{4}\left(\sqrt{3} T_{8 R}-T_{3 R}\right)+X^{\prime}
$$

and the electric charge is $Q=T_{3 L}+Y$, all of which have the usual form.

\subsubsection{Case 3: $w, w^{\prime} \sim \Lambda$}

Another possible breaking pattern takes place when assuming that the symmetry breaking of the left-right and $\mathrm{SU}(3)_{L}$ symmetry occurs at the same scale, i.e. $w, w^{\prime} \sim \Lambda$. Therefore, we have only one new physics scale and the gauge symmetry is directly broken down to that of the SM as,

$$
\begin{gathered}
\mathrm{SU}(3)_{C} \otimes \mathrm{SU}(3)_{L} \otimes \mathrm{SU}(3)_{R} \otimes \mathrm{U}(1)_{X} \\
\downarrow \Lambda, w, w^{\prime} \\
\mathrm{SU}(3)_{C} \otimes \mathrm{SU}(2)_{L} \otimes \mathrm{U}(1)_{Y} \otimes W_{P} \\
\downarrow u, u^{\prime} \\
\mathrm{SU}(3)_{C} \otimes \mathrm{U}(1)_{Q} \otimes W_{P} .
\end{gathered}
$$

Here, $W_{P}$ is the residual discrete gauge symmetry preserved by all VEVs and has the form obtained above.

In summary, regardless of symmetry breaking scheme adopted, they all lead to the residual conserved $W$-parity, $W_{P}=(-1)^{3(B-L)+2 s}$, with $B-L=2\left[\beta\left(T_{8 L}+T_{8 R}\right)+X\right]$. In this way, the matter parity is a direct consequence of the gauge group and as we shall see, it naturally leads to the existence of stable dark matter particles.

The transformation properties of the particles of the model under $B-L$ number and $W$-parity are collected in table 1 . Notice that the $B-L$ charge for the new particles depends on their electric charge, i.e. on the basic electric charge parameter $q$, with $W$ parity values $P^{ \pm} \equiv(-1)^{ \pm(6 q+1)}$. When the new particles have ordinary electric charges $q=m / 3$ for $m$ integer, they are $W$-odd, $P^{ \pm}=-1$, analogously to superparticles in supersymmetry. Generally, assuming that $q \neq(2 m-1) / 6, W$-parity is nontrivial, with $P^{ \pm} \neq 1$ and $\left(P^{+}\right)^{\dagger}=P^{-}$. Such new particles, denoted as $W$-particles in what follows, 


\begin{tabular}{|c|ccccccccccc|}
\hline Particle & $\nu_{a}$ & $e_{a}$ & $N_{a}$ & $u_{a}$ & $d_{a}$ & $J_{\alpha}$ & $J_{3}$ & $\phi_{11}^{0}$ & $\phi_{12}^{+}$ & $\phi_{13}^{-q}$ & $\phi_{21}^{-}$ \\
\hline$B-L$ & -1 & -1 & $2 q$ & $\frac{1}{3}$ & $\frac{1}{3}$ & $-\frac{2(1+3 q)}{3}$ & $\frac{2(2+3 q)}{3}$ & 0 & 0 & $-(1+2 q)$ & 0 \\
\hline$W_{P}$ & 1 & 1 & $P^{+}$ & 1 & 1 & $P^{-}$ & $P^{+}$ & 1 & 1 & $P^{-}$ & 1 \\
\hline Particle & $\phi_{31}^{q}$ & $\phi_{32}^{1+q}$ & $\phi_{33}^{0}$ & $\phi_{22}^{0}$ & $\phi_{23}^{-1-q}$ & $\chi_{1}^{(-q)}$ & $\chi_{2}^{-(q+1)}$ & $\chi_{3}^{0}$ & $\sigma_{11}^{0}$ & $\sigma_{12}^{-}$ & $\sigma_{13}^{q}$ \\
\hline$B-L$ & $(1+2 q)$ & $(1+2 q)$ & 0 & 0 & $-(1+2 q)$ & $-(1+2 q)$ & $-(1+2 q)$ & 0 & -2 & -2 & $-1+2 q$ \\
\hline$W_{P}$ & $P^{+}$ & $P^{+}$ & 1 & 1 & $P^{-}$ & $P^{-}$ & $P^{-}$ & 1 & 1 & 1 & $P^{+}$ \\
\hline Particle & $\sigma_{22}^{--}$ & $\sigma_{23}^{q-1}$ & $\sigma_{33}^{2 q}$ & $A$ & $Z_{L, R}$ & $Z_{L, R}^{\prime}$ & $W_{L, R}^{+}$ & $X_{L, R}^{q}$ & $X_{L, R}^{-q}$ & $Y_{L, R}^{q+1}$ & $Y_{L, R}^{-(q+1)}$ \\
\hline$B-L$ & -2 & $-1+2 q$ & $4 q$ & 0 & 0 & 0 & 0 & $1+2 q$ & $-(1+2 q)$ & $1+2 q$ & $-(1+2 q)$ \\
\hline$W_{P}$ & 1 & $P^{+}$ & $P^{+}$ & 1 & 1 & 1 & 1 & $P^{+}$ & $P^{-}$ & $P^{+}$ & $P^{-}$ \\
\hline
\end{tabular}

Table 1. The $B-L$ number and $W$-parity of the model particles, with $P^{ \pm} \equiv(-1)^{ \pm(6 q+1)}$.

have different $B-L$ numbers than those of the standard model. Recall that $W$-parity is only trivial for $q=(2 m-1) / 6= \pm 1 / 6, \pm 1 / 2, \pm 5 / 6, \pm 7 / 6, \cdots$, values not studied in this work as they require fractional charges for the new leptons.

Since the $W$-charged and SM particles are unified within the gauge multiplets, $W$ parity separates them into two classes,

- Normal particles with $W_{P}=1$ : consist on all SM particles plus extra new fields. Explicitly, the particles belonging to this class are the fermions, $\nu_{a}, e_{a}, u_{a}, d_{a}$, the scalars, $\phi_{11}^{0}, \phi_{12}^{ \pm}, \phi_{21}^{ \pm}, \phi_{22}^{0}, \phi_{33}^{0}, \chi_{3}^{0}, \sigma_{11}^{0}, \sigma_{12}^{ \pm}, \sigma_{22}^{ \pm \pm}, \sigma_{33}^{ \pm 2 q}$, the gauge bosons, $A, Z_{L, R}, Z_{L, R}^{\prime}$, and the gluon.

- $W$-particles with $W_{P}=P^{+}$or $P^{-}$: includes the new leptons and quarks, $N_{a}, J_{a}$, the new scalars, $\phi_{13}^{ \pm q}, \phi_{23}^{ \pm(1+q)}, \phi_{31}^{ \pm q}, \phi_{32}^{ \pm(1+q)}, \chi_{1}^{ \pm q}, \chi_{2}^{ \pm(q+1)} \sigma_{13}^{ \pm q}, \sigma_{23}^{ \pm(q-1)}$, and the new nonHermitian gauge bosons, $X_{L, R}^{ \pm q}, Y_{L, R}^{ \pm(q+1)}$.

It can be easily shown that $W$-particles always appear in pairs in interactions, similarly to superparticles in supersymmetry. Indeed, consider an interaction that includes $x P^{+}$fields and $y P^{-}$-fields. The $W$-parity conservation implies $(-1)^{(6 q+1)(x-y)}=1$ for arbitrary $q$ which is satisfied only if $x=y$. Hence, the fields $P^{+}$and $P^{-}$are always coupled in pairs. The lightest $W$-particle (often called LWP) cannot decay due to the $W$-parity conservation. Thus, if the lightest $W$-particle carries no electrical and color charges, it can be identified as a dark matter candidate.

From table 1, the colorless $W$-particles have electrical charges $\pm q, \pm(1+q), \pm(q-1)$, and therefore three dark matter models can be built, corresponding to $q=0, \pm 1 .{ }^{3}$ The model $q=0$ includes three dark matter candidates, namely, a lepton as the lightest mixture of $N_{a}^{0}$, a scalar as the combination of $\phi_{13}^{0}, \phi_{31}^{0}, \chi_{1}^{0}, \sigma_{13}^{0}$, and a gauge boson from the mixing of $X_{L, R}^{0}$. The model $q=-1$ contains two dark matter candidates: a scalar composed of $\phi_{23}^{0}, \phi_{32}^{0}, \chi_{2}^{0}$ and a gauge boson from the lightest mixture of $Y_{L, R}^{0}$. Lastly, the model $q=1$ has only one dark matter candidate: the scalar field $\sigma_{23}^{0}$.

\footnotetext{
${ }^{3}$ The $q=1$ case might be ruled out in the manifest left-right model [38].
} 
Before closing this section, it is important to notice that the fundamental field $\sigma_{33}^{2 q}$, carrying $W$-parity $\left(P^{+}\right)^{2}$, leads to self-interactions among three $W$-fields, if it transforms nontrivially under this parity. However, its presence does not alter the results and conclusions given below. See [39] for a proof.

\section{$3 \quad$ Identifying physical states and masses}

The Lagrangian of the model takes the form, $\mathcal{L}=\mathcal{L}_{\text {gauge }}+\mathcal{L}_{\text {Yukawa }}-V$, where the first term contains all kinetic terms plus gauge interactions. The second term includes Yukawa interactions, obtained by

$$
\begin{aligned}
\mathcal{L}_{\text {Yukawa }}= & x_{a b} \bar{\psi}_{a R}^{c} \sigma_{R}^{\dagger} \psi_{b R}+x_{a b}^{\prime} \bar{\psi}_{a L}^{c} \sigma_{L}^{\dagger} \psi_{b L}+y_{a b} \bar{\psi}_{a L} \phi \psi_{b R}+z_{33} \bar{Q}_{3 L} \phi Q_{3 R}+z_{\alpha \beta} \bar{Q}_{\alpha L} \phi^{*} Q_{\beta R} \\
& +\frac{t_{3 \alpha}}{M} \bar{Q}_{3 L} \phi \chi^{*} Q_{\alpha R}+\frac{t_{\alpha 3}}{M} \bar{Q}_{\alpha L} \phi^{*} \chi Q_{3 R}+H . c .
\end{aligned}
$$

where $M$ is a new physics scale that defines the effective interactions required to generate a consistent CKM matrix. The scalar potential is $V=V_{\phi}+V_{\chi}+V_{\sigma}+V_{\text {mix }}$, where

$$
\begin{aligned}
V_{\phi}= & \mu_{\phi}^{2} \operatorname{Tr}\left(\phi^{\dagger} \phi\right)+\lambda_{1}\left[\operatorname{Tr}\left(\phi^{\dagger} \phi\right)\right]^{2}+\lambda_{2} \operatorname{Tr}\left[\left(\phi^{\dagger} \phi\right)^{2}\right], \\
V_{\chi}= & \mu_{\chi}^{2} \chi^{\dagger} \chi+\lambda\left(\chi^{\dagger} \chi\right)^{2}, \\
V_{\sigma}= & \mu_{\sigma}^{2} \operatorname{Tr}\left(\sigma^{\dagger} \sigma\right)+\kappa_{1}\left[\operatorname{Tr}\left(\sigma^{\dagger} \sigma\right)\right]^{2}+\kappa_{2} \operatorname{Tr}\left[\left(\sigma^{\dagger} \sigma\right)^{2}\right], \\
V_{\text {mix }}= & \zeta_{1} \chi^{\dagger} \chi \operatorname{Tr}\left(\phi^{\dagger} \phi\right)+\zeta_{2} \operatorname{Tr}\left(\phi^{\dagger} \phi\right) \operatorname{Tr}\left(\sigma^{\dagger} \sigma\right)+\zeta_{3} \operatorname{Tr}\left(\phi^{\dagger} \phi \sigma \sigma^{\dagger}\right)+\zeta_{4} \chi^{\dagger} \chi \operatorname{Tr}\left(\sigma^{\dagger} \sigma\right) \\
& +\zeta_{5} \chi^{\dagger} \sigma \sigma^{\dagger} \chi+\zeta_{6} \chi^{\dagger} \phi^{\dagger} \phi \chi+\left(f \epsilon^{i j k} \epsilon_{\alpha \beta \gamma} \phi_{i}^{\alpha} \phi_{j}^{\beta} \phi_{k}^{\gamma}+\text { H.c. }\right) .
\end{aligned}
$$

We see that $\phi$ has trilinear couplings. An $\mathrm{SU}(2)_{L}$ doublet contained in $\phi$ can be made heavy by taking $f$ at the new physics scale. The remaining Higgs doublet in $\phi$ is light and lies in the weak scale, as shown below. If another bi-fundamental field $\rho$ is introduced in this minimal framework, coupling the third quark generation to the first two, there are no such soft-terms for arbitrary values of the $\beta$ parameter, since its $X$-charge is nonzero. Thus, both the Higgs doublets contained in $\rho$ would be light as their VEVs are in the weak scale. In order to avoid light scalars, the triplet $\chi$ is included in this work instead of $\rho$ in order to generate viable quark masses and mixings.

\subsection{Fermion sector}

After spontaneous symmetry breaking, the fermions receive their masses via the Yukawa Lagrangian (3.1). For the up-type quarks and down-type quarks, the corresponding mass matrices are given by

$$
M_{u}=-\frac{1}{\sqrt{2}}\left(\begin{array}{ccc}
z_{11} u^{\prime} & z_{12} u^{\prime} & -\frac{t_{13} u^{\prime} w^{\prime}}{\sqrt{2} M} \\
z_{21} u^{\prime} & z_{22} u^{\prime} & -\frac{t_{23} u^{\prime} w^{\prime}}{\sqrt{2} M} \\
\frac{t_{31} u w^{\prime}}{\sqrt{2} M} & \frac{t_{32} u w^{\prime}}{\sqrt{2} M} & z_{33} u
\end{array}\right), \quad M_{d}=-\frac{1}{\sqrt{2}}\left(\begin{array}{ccc}
z_{11} u & z_{12} u & -\frac{t_{13} u w^{\prime}}{\sqrt{2} M} \\
z_{21} u & z_{22} u & -\frac{t_{23} u w^{\prime}}{\sqrt{2} M} \\
\frac{t_{31} u^{\prime} w^{\prime}}{\sqrt{2} M} & \frac{t_{32} u^{\prime} w^{\prime}}{\sqrt{2} M} & z_{33} u^{\prime}
\end{array}\right) .
$$

The ordinary quarks obtain consistent masses at the weak scale, $u, u^{\prime}$. The new physics or cut-off scale can be taken as at the largest breaking scale, $M \sim w^{\prime}$. The scale $\mathrm{M}$ 
characterizing the non-renormalizable interaction is responsible for generating $V_{u b}, V_{c b}$, as well as quark $\mathrm{CP}$ violation, as required.

The exotic quark, $J_{3}$, is a physical field by itself, with mass, $m_{J_{3}}=-\frac{z_{33} w}{\sqrt{2}}$, which is heavy, lying at the new physics regime. The two remaining exotic quarks, $J_{\alpha}(\alpha=1,2)$, mix via a mass matrix,

$$
M_{J_{\alpha}}=-\frac{1}{\sqrt{2}}\left(\begin{array}{cc}
z_{11} w & z_{12} w \\
z_{21} w & z_{22} w
\end{array}\right),
$$

and are both heavy, at the new physics regime too.

The mass matrix elements for the charged leptons,

$$
\left[M_{e}\right]_{a b}=-\frac{1}{\sqrt{2}} y_{a b} u^{\prime}
$$

belong to the weak regime as usual. In contrast, the new leptons, $N_{a}$, have large masses dictated by the mass matrix

$$
\left[M_{N}\right]_{a b}=-\frac{1}{\sqrt{2}} y_{a b} w
$$

Neutrinos have both Dirac and Majorana masses. The mass matrix in the $\left(\begin{array}{ll}\nu_{L} & \nu_{R}^{c}\end{array}\right)$ basis can be written as

$$
M_{\nu}=\left(\begin{array}{ll}
M_{L} & M_{D} \\
M_{D}^{T} & M_{R}
\end{array}\right),
$$

where $M_{L}, M_{D}, M_{R}$ are $3 \times 3$ mass matrices, given by

$$
\left[M_{D}\right]_{a b}=-\frac{1}{\sqrt{2}} y_{a b} u, \quad\left[M_{L}\right]_{a b}=-\sqrt{2} x_{a b}^{\prime} v_{L}, \quad\left[M_{R}\right]_{a b}=-\sqrt{2} x_{a b} \Lambda,
$$

with $\left\langle\sigma_{L 11}^{0}\right\rangle=v_{L} / \sqrt{2}$. As $v_{L} \ll u \ll \Lambda$, the mass matrix (3.11) provides a realization of the full seesaw mechanism, producing small masses for the light neutrinos $\sim \nu_{L}$,

$$
m_{\nu}=M_{L}-M_{D} M_{R}^{-1} M_{D}^{T} \sim u^{2} / \Lambda-v_{L},
$$

and large masses for the mostly right-handed neutrinos $\sim \nu_{R}$, of order $M_{R}$.

\subsection{Scalar sector}

Since $W$-parity is conserved, only the neutral fields carrying $W_{P}=1$ can develop the VEVs given in (2.12). We expand the fields around their VEVs as

$$
\begin{aligned}
\sigma & =\left(\begin{array}{ccc}
\frac{\Lambda+S_{1}+i A_{1}}{\sqrt{2}} & \frac{\sigma_{12}^{-}}{\sqrt{2}} & \frac{\sigma_{13}^{q}}{\sqrt{2}} \\
\frac{\sigma_{12}^{-}}{\sqrt{2}} & \sigma_{22}^{-} & \frac{\sigma_{23}^{q-1}}{\sqrt{2}} \\
\frac{\sigma_{13}^{q}}{\sqrt{2}} & \frac{\sigma_{23}^{q-1}}{\sqrt{2}} & \sigma_{33}^{2 q}
\end{array}\right), \\
\phi & =\left(\begin{array}{ccc}
\frac{u+S_{2}+i A_{2}}{\sqrt{2}} & \phi_{12}^{+} & \phi_{13}^{-q} \\
\phi_{21}^{-} & \frac{u^{\prime}+S_{3}+i A_{3}}{\sqrt{2}} & \phi_{23}^{-(q+1)} \\
\phi_{31}^{q} & \phi_{32}^{q+1} & \frac{w+S_{4}+i A_{4}}{\sqrt{2}}
\end{array}\right), \\
\chi & =\left(\begin{array}{c}
\chi_{1}^{-q} \\
\chi_{2}^{-(q+1)} \\
\frac{w^{\prime}+S_{5}+i A_{5}}{\sqrt{2}}
\end{array}\right) .
\end{aligned}
$$


The scalar potential can be written as $V=V_{\text {min }}+V_{\text {linear }}+V_{\text {mass }}+V_{\text {int }}$, where $V_{\text {min }}$ is independent of the fields, and all interactions are grouped into $V_{\text {int }}$. $V_{\text {linear }}$ contains all the terms that depend linearly on the fields, and the gauge invariance requires,

$$
\begin{aligned}
2 \mu_{\sigma}^{2}+\left(u^{\prime 2}+w^{2}\right) \zeta_{2}+u^{\prime 2}\left(\zeta_{2}+\zeta_{3}\right)+w^{\prime 2} \zeta_{4}+2\left(\kappa_{1}+\kappa_{2}\right) \Lambda^{2} & =0, \\
2 \mu_{\phi}^{2}+6 \sqrt{2} f \frac{u^{\prime}}{u} w+2 \lambda_{1}\left(u^{2}+u^{\prime 2}+w^{2}\right)+\left(2 \lambda_{2} u^{2}+\zeta_{1} w^{\prime 2}+\left(\zeta_{2}+\zeta_{3}\right) \Lambda^{2}\right) & =0, \\
2 \mu_{\phi}^{2}+6 \sqrt{2} f \frac{u}{u^{\prime}} w+2\left(\lambda_{1}+\lambda_{2}\right) u^{\prime 2}+\left(2 \lambda_{1}\left(u^{\prime 2}+w^{2}\right)+\zeta_{1} w^{\prime 2}+\zeta_{2} \Lambda^{2}\right) & =0, \\
2 \mu_{\phi}^{2}+6 \sqrt{2} f \frac{u u^{\prime}}{w}+2 \lambda_{1}\left(u^{2}+u^{\prime 2}\right)+2\left(\lambda_{1}+\lambda_{2}\right) w^{2}+\left(\zeta_{1}+\zeta_{6}\right) w^{\prime 2}+\zeta_{2} \Lambda^{2} & =0, \\
2 \mu_{\chi}^{2}+2 \lambda w^{\prime 2}+\zeta_{1}\left(u^{2}+u^{\prime 2}+w^{2}\right)+\zeta_{6} w^{2}+\zeta_{4} \Lambda^{2} & =0 .
\end{aligned}
$$

$V_{\text {mass }}$ consists of the terms that quadratically depend on the fields, and can be furhter decomposed as $V_{\text {mass }}=V_{\text {mass }}^{A}+V_{\text {mass }}^{S}+V_{\text {mass }}^{\text {singly-charged }}+V_{\text {mass }}^{\text {doubly-charged }}+V_{\text {mass }}^{q \text {-charged }}+V_{\text {mass }}^{(q+1) \text {-charged }}$ $+V_{\text {mass }}^{(q-1) \text {-charged }}+V_{\text {mass }}^{2 q-\text { charged }}$, which are listed in appendix $\mathrm{A}$.

The first mass term includes all pseudo-scalars $A_{1}, A_{2}, A_{3}, A_{4}, A_{5}$. From appendix A, we see that $A_{1}, A_{5}$ are massless and can be identified to the Goldstone bosons of the righthanded neutral gauge bosons, $\mathcal{Z}_{R}, \mathcal{Z}_{R}^{\prime}$, respectively. The remaining fields $A_{2}, A_{3}, A_{4}$ mix, but their mass matrix produces only one physical pseudo-scalar field with mass

$$
\begin{aligned}
\mathcal{A} & =\frac{1}{\sqrt{u^{2} w^{2}+u^{\prime 2} w^{2}+u^{2} u^{\prime 2}}}\left[u^{\prime} w A_{2}+u w A_{3}+u^{\prime} u A_{4}\right], \\
m_{\mathcal{A}}^{2} & =\frac{\left[u^{\prime 2} w^{2}+u^{2}\left(u^{\prime 2}+w^{2}\right)\right]\left[2 \lambda_{2}\left(u^{\prime 2}-w^{2}\right)-\zeta_{6} w^{\prime 2}\right]}{2 u^{2}\left(w^{2}-u^{\prime 2}\right)},
\end{aligned}
$$

which is heavy, at the $w, w^{\prime}$ scale. The remaining fields are massless and orthogonal to $\mathcal{A}$

$$
\begin{aligned}
G_{\mathcal{Z}_{L}} & =\sqrt{\frac{u^{2}\left(w^{2}+u^{\prime 2}\right)}{w^{2} u^{\prime 2}+u^{2}\left(w^{2}+u^{\prime 2}\right)}}\left\{-A_{2}+\frac{u^{\prime} w^{2}}{u\left(w^{2}+u^{\prime 2}\right)} A_{3}+\frac{u^{\prime 2} w}{u\left(w^{2}+u^{\prime 2}\right)} A_{4}\right\}, \\
G_{\mathcal{Z}_{L}^{\prime}} & =\frac{u^{\prime}}{\sqrt{w^{2}+u^{\prime 2}}} A_{3}-\frac{w}{\sqrt{w^{2}+u^{\prime 2}}} A_{4},
\end{aligned}
$$

and can be identified with the Goldstone bosons of the neutral boson $\mathcal{Z}_{L}$, analogous to the SM $Z$ boson, and the new neutral gauge boson $\mathcal{Z}_{L}^{\prime}$.

The $V_{\text {mass }}^{S}$ term contains all the mass terms of the scalar fields, $S_{1}, S_{2}, S_{3}, S_{4}, S_{5}$, as shown in appendix A. The five scalars mix through a $5 \times 5$ matrix. In general, it is not easy to find the eigenstates. However, using the fact that $u, v \ll w^{\prime}, w, \Lambda$, one can diagonalize the mass matrix perturbatively. At leading order, this matrix yields one massless scalar field, $H_{1}=\frac{1}{\sqrt{u^{2}+u^{\prime 2}}}\left(u S_{2}+u^{\prime} S_{3}\right)$, and a massive scalar field, $H_{2}=\frac{1}{\sqrt{u^{2}+u^{\prime 2}}}\left(u^{\prime} S_{2}-u S_{3}\right)$, with $m_{H_{2}}^{2}=-\frac{u^{2}+u^{\prime 2}}{2 u^{2}}\left(\zeta_{6} w^{\prime 2}+2 \lambda_{2} w^{2}\right)$. The $H_{1}$ field obtains a mass at next-to-leading order, $m_{H_{1}} \simeq O\left(u, u^{\prime}\right)$, and is identified with the standard model Higgs boson. The remaining fields, $\left(S_{1}, S_{4}, S_{5}\right)$, are heavy and mixed among themselves via a $3 \times 3$ matrix. In the limit, 
$\Lambda \gg w, w^{\prime}$, the corresponding physical fields have masses given by

$$
\begin{aligned}
H_{3}= & S_{1}, \quad m_{H_{3}}^{2}=\frac{1}{2}\left(\kappa_{1}+\kappa_{2}\right) \Lambda^{2}, \\
H_{4}= & c_{H} S_{4}-s_{H} S_{5}, \\
m_{H_{4}}^{2}= & \frac{1}{2}\left\{\left(\lambda_{1}+\lambda_{2}\right) w^{2}+\lambda w^{\prime 2}-\frac{\left(\zeta_{2}^{2} w^{2}+\zeta_{4}^{2} w^{\prime 2}\right)}{4\left(\kappa_{1}+\kappa_{2}\right)}\right. \\
& \left.+\sqrt{\left[\left(\lambda_{1}+\lambda_{2}\right) w^{2}-\lambda w^{\prime 2}+\frac{\zeta_{4}^{2} w^{\prime 2}-\zeta_{2}^{2} w^{2}}{4\left(\kappa_{1}+\kappa_{2}\right)}\right]^{2}+\frac{1}{4} w^{2} w^{\prime 2}\left(\frac{\zeta_{2} \zeta_{4}}{\kappa_{1}+\kappa_{2}}-2\left(\zeta_{1}+\zeta_{6}\right)\right)^{2}}\right\}, \\
H_{5}= & s_{H} S_{4}+c_{H} S_{5}, \\
m_{H_{5}}^{2}= & \frac{1}{2}\left\{\left(\lambda_{1}+\lambda_{2}\right) w^{2}+\lambda w^{\prime 2}-\frac{\left(\zeta_{2}^{2} w^{2}+\zeta_{4}^{2} w^{\prime 2}\right)}{4\left(\kappa_{1}+\kappa_{2}\right)}\right]^{2} \\
& \left.-\sqrt{\left[\left(\lambda_{1}+\lambda_{2}\right) w^{2}-\lambda w^{\prime 2}+\frac{\zeta_{4}^{2} w^{\prime 2}-\zeta_{2}^{2} w^{2}}{4\left(\kappa_{1}+\kappa_{2}\right)}\right]^{2}+\frac{1}{4} w^{2} w^{\prime 2}\left(\frac{\zeta_{2} \zeta_{4}}{\kappa_{1}+\kappa_{2}}-2\left(\zeta_{1}+\zeta_{6}\right)\right)^{2}}\right\},
\end{aligned}
$$

where the mixing angle $\theta_{H}$ is defined by the relation

$$
t_{2 \theta_{H}}=\frac{w w^{\prime}\left\{-\frac{\zeta_{2} \zeta_{4}}{\kappa_{1}+\kappa_{2}}+2\left(\zeta_{1}+\zeta_{6}\right)\right\}}{2\left\{-\left(\lambda_{1}+\lambda_{2}\right) w^{2}+\lambda w^{2}+\frac{\zeta_{2}^{2} w^{2}-\zeta_{4}^{2} w^{\prime 2}}{4\left(\kappa_{1}+\kappa_{2}\right)}\right\}} .
$$

On the other hand, if one assumes that instead the hierarchy $w, w^{\prime}>\Lambda$ holds, the masses and mixing of the heavy states, $\left(H_{4}, H_{5}\right)$ change accordingly to

$$
\begin{aligned}
t_{2 \theta_{H}} & =\frac{w w^{\prime}\left(\zeta_{1}+\zeta_{6}\right)}{\lambda w^{\prime 2}-\left(\lambda_{1}+\lambda_{2}\right) w^{2}}, \\
m_{H_{4}}^{2} & =\frac{1}{2}\left\{\left(\lambda_{1}+\lambda_{2}\right) w^{2}+\lambda w^{\prime 2}+\sqrt{\left[\left(\lambda_{1}+\lambda_{2}\right) w^{2}-\lambda w^{\prime 2}\right]^{2}+w^{2} w^{\prime 2}\left(\zeta_{1}+\zeta_{6}\right)^{2}}\right\}, \\
m_{H_{5}}^{2} & =\frac{1}{2}\left\{\left(\lambda_{1}+\lambda_{2}\right) w^{2}+\lambda w^{\prime 2}-\sqrt{\left[\left(\lambda_{1}+\lambda_{2}\right) w^{2}-\lambda w^{\prime 2}\right]^{2}+w^{2} w^{\prime 2}\left(\zeta_{1}+\zeta_{6}\right)^{2}}\right\} .
\end{aligned}
$$

Turning now to the singly-charged Higgs fields, we have three fields plus their conjugates. The mass matrix extracted from (A.3) yields four massless fields, which can be identified to the Goldstone bosons of the $W_{L, R}^{ \pm}$gauge bosons,

$$
\begin{aligned}
& G_{W_{L}}^{ \pm}=\frac{1}{\sqrt{u^{2}+u^{\prime 2}}}\left\{u^{\prime} \phi_{12}^{ \pm}-u \phi_{21}^{ \pm}\right\}, \\
& G_{W_{R}}^{ \pm}=\frac{1}{\sqrt{1+\frac{u^{\prime 2}}{u^{2}}+\frac{2\left(u^{2}+u^{\prime 2}\right)^{2} \Lambda^{2}}{u^{2}\left(u^{2}-u^{\prime 2}\right)^{2}}}}\left\{\frac{\sqrt{2}\left(u^{2}+u^{\prime 2}\right) \Lambda}{u\left(u^{2}-u^{\prime 2}\right)} \sigma_{12}^{ \pm}+\phi_{12}^{ \pm}+\frac{u^{\prime}}{u} \phi_{21}^{ \pm}\right\},
\end{aligned}
$$

and two singly-charged massive Higgs fields with corresponding masses

$$
\begin{aligned}
H^{ \pm} & =\frac{\sqrt{2} u \Lambda}{\sqrt{\left(u^{2}-u^{\prime 2}\right)^{2}+2 \Lambda^{2}\left(u^{2}+u^{\prime 2}\right)}}\left\{\frac{u^{\prime 2}-u^{2}}{\sqrt{2} u \Lambda} \sigma_{12}^{ \pm}+\phi_{12}^{ \pm}+\frac{u^{\prime}}{u} \phi_{21}^{ \pm}\right\}, \\
m_{H^{ \pm}}^{2} & =\frac{\left\{2 \lambda_{2}\left(u^{2}-w^{2}\right)\left(u^{\prime 2}-w^{2}\right)+\zeta_{6} w^{2} w^{\prime 2}\right\}\left\{\left(u^{2}-u^{\prime 2}\right)^{2}+2\left(u^{2}+u^{\prime 2}\right) \Lambda^{2}\right\}}{4 u^{2}\left(u^{\prime 2}-w^{2}\right) \Lambda^{2}} .
\end{aligned}
$$


There is only one doubly-charged Higgs field, $\sigma_{22}^{ \pm \pm}$, and is physical by itself, with mass

$$
m_{\sigma_{22}}^{2}=\frac{\left(u^{2}-u^{\prime 2}\right)^{2}\left[2 \lambda_{2}\left(u^{2}-w^{2}\right)\left(u^{\prime 2}-w^{2}\right)+\zeta_{6} w^{2} w^{\prime 2}\right]+2 \kappa_{2} u^{2}\left(w^{2}-u^{\prime 2}\right) \Lambda^{4}}{2 u^{2} \Lambda^{2}\left(u^{\prime 2}-w^{2}\right)} .
$$

For $q$-charged scalars, $V_{\text {mass }}^{q \text {-charged }}$ contains the fields, $\phi_{13}^{ \pm q}, \phi_{31}^{ \pm q}, \sigma_{13}^{ \pm q}, \chi_{1}^{ \pm q}$, as shown in appendix A. The spectrum in this sector includes four massless Goldstone bosons of the new gauge bosons $X_{L, R}^{ \pm q}$,

$$
\begin{aligned}
G_{X_{L}}^{ \pm q}= & \frac{1}{\sqrt{u^{4}+w^{4}+u^{2}\left(w^{\prime 2}+2 \Lambda^{2}-2 w^{2}\right)}}\left\{\left(w^{2}-u^{2}\right) \phi_{13}^{ \pm q}-\sqrt{2} u \Lambda \sigma_{13}^{ \pm q}+u w^{\prime} \chi_{1}^{ \pm q}\right\} \\
G_{X_{R}}^{ \pm q}= & \frac{1}{\sqrt{\left(u^{4}+w^{4}+u^{2}\left(w^{\prime 2}+2 \Lambda^{2}-2 w^{2}\right)\right)\left(u^{4}+u^{2}\left(w^{\prime 2}+2 \Lambda^{2}-2 w^{2}\right)+w^{2}\left(w^{2}+w^{\prime 2}+2 \Lambda^{2}\right)\right)}} \\
& \times\left\{u w\left(w^{\prime 2}+2 \Lambda^{2}\right) \phi_{13}^{ \pm q}+\left(-u^{4}-w^{4}+u^{2}\left(2 w^{2}-2 \Lambda^{2}-w^{\prime 2}\right)\right) \phi_{31}^{ \pm q}+\sqrt{2} w \Lambda\left(w^{2}-u^{2}\right) \sigma_{13}^{ \pm q}\right. \\
& \left.+\left(u^{2}-w^{2}\right) w^{\prime} w \chi_{1}^{ \pm q}\right\} .
\end{aligned}
$$

The remaining fields are massive. In the limit, $\Lambda, w, w^{\prime} \gg u, u^{\prime}$, their physical states are

$$
\begin{aligned}
\mathcal{H}_{1}^{ \pm q} \simeq & \frac{c_{\theta_{q}}}{\sqrt{w^{\prime 2}+2 \Lambda^{2}}}\left\{w^{\prime} \sigma_{13}^{ \pm q}+\sqrt{2} \Lambda \chi_{1}^{ \pm q}\right\} \\
& -\frac{s_{\theta_{q}}}{\sqrt{\left(w^{2}+2 \Lambda^{2}\right)\left(w^{2}+w^{\prime 2}+2 \Lambda^{2}\right)}}\left\{\left(w^{\prime 2}+2 \Lambda^{2}\right) \phi_{31}^{ \pm q}+\sqrt{2} w \Lambda \sigma_{13}^{ \pm q}-w w^{\prime} \chi_{1}^{ \pm q}\right\}, \\
\mathcal{H}_{2}^{ \pm q} \simeq & \frac{s_{\theta_{q}}}{\sqrt{w^{\prime 2}+2 \Lambda^{2}}}\left\{w^{\prime} \sigma_{13}^{ \pm q}+\sqrt{2} \Lambda \chi_{1}^{ \pm q}\right\} \\
& +\frac{c_{\theta_{q}}}{\sqrt{\left(w^{2}+2 \Lambda^{2}\right)\left(w^{2}+w^{\prime 2}+2 \Lambda^{2}\right)}}\left\{\left(w^{\prime 2}+2 \Lambda^{2}\right) \phi_{31}^{ \pm q}+\sqrt{2} w \Lambda \sigma_{13}^{ \pm q}-w w^{\prime} \chi_{1}^{ \pm q}\right\},
\end{aligned}
$$

with masses

$$
\begin{aligned}
m_{\mathcal{H}_{1}}^{2}= & \frac{1}{4 \Lambda^{2}}\left\{w^{\prime 2}\left(\zeta_{5}-2 t_{u}^{2} \zeta_{6}\right) \Lambda^{2}+2 \zeta_{5} \Lambda^{4}+\zeta_{6} w^{2}\left(w^{\prime 2}-t_{u}^{2} w^{\prime 2}-2 \Lambda^{2}\right)-2 \lambda_{2}\left(t_{u}^{2}-1\right) w^{2}\left(w^{2}+2 \Lambda^{2}\right)\right\} \\
& +\frac{1}{2 \Lambda\left(w^{\prime 2}+2 \Lambda^{2}\right)} \sqrt{2 w^{2} w^{\prime 2}\left(w^{2}+w^{\prime 2}+2 \Lambda^{2}\right)\left[\left(t_{u}^{2}-1\right)\left(2 \lambda_{2} w^{2}+\zeta_{6} w^{\prime 2}\right)-2 \zeta_{6} \Lambda^{2}\right]^{2}+\epsilon_{H^{ \pm}} q}, \\
m_{\mathcal{H}_{2}}^{2}= & \frac{1}{4 \Lambda^{2}}\left\{w^{\prime 2}\left(\zeta_{5}-2 t_{u}^{2} \zeta_{6}\right) \Lambda^{2}+2 \zeta_{5} \Lambda^{4}+\zeta_{6} w^{2}\left(w^{\prime 2}-t_{u}^{2} w^{\prime 2}-2 \Lambda^{2}\right)-2 \lambda_{2}\left(t_{u}^{2}-1\right) w^{2}\left(w^{2}+2 \Lambda^{2}\right)\right\} \\
& -\frac{1}{2 \Lambda\left(w^{\prime 2}+2 \Lambda^{2}\right)} \sqrt{2 w^{2} w^{\prime 2}\left(w^{2}+w^{\prime 2}+2 \Lambda^{2}\right)\left[\left(t_{u}^{2}-1\right)\left(2 \lambda_{2} w^{2}+\zeta_{6} w^{\prime 2}\right)-2 \zeta_{6} \Lambda^{2}\right]^{2}+\epsilon_{H^{ \pm} q}}
\end{aligned}
$$

where

$$
\begin{aligned}
t_{\theta_{q}}= & \frac{2 \sqrt{2} w w^{\prime} \Lambda\left(w^{2}+2 \Lambda^{2}\right) \sqrt{w^{2}+w^{\prime 2}+2 \Lambda^{2}}\left\{2 \lambda_{2}\left(t_{u}^{2}-1\right) w^{2}+\zeta_{6}\left(\left(t_{u}^{2}-1\right) w^{2}-2 \Lambda^{2}\right)\right\}}{\epsilon_{H^{ \pm} q}}, \\
\epsilon_{H^{ \pm} q}= & \left(w^{\prime 2}+2 \Lambda^{2}\right)\left[\Lambda^{2}\left(w^{\prime 2}+2 \Lambda^{2}\right)\left(w^{\prime 2}\left(\zeta_{5}+2 t_{u}^{2} \zeta_{6}\right)+2 \zeta_{5} \Lambda^{2}\right)\right. \\
& +\zeta_{6} w^{2}\left(w^{\prime 4}\left(1-t_{u}^{2}\right)+2 t_{u}^{2} w^{\prime 2} \Lambda^{2}-4 \Lambda^{4}\right) \\
& \left.-2 \lambda_{2}\left(t_{u}^{2}-1\right) w^{2}\left(w^{2} w^{\prime 2}-2\left(w^{2}+w^{\prime 2}\right) \Lambda^{2}-4 \Lambda^{4}\right)\right] .
\end{aligned}
$$


$V_{\text {mass }}^{(q+1) \text {-charged }}$ contains the mixing terms of $\phi_{23}^{ \pm(q+1)}, \phi_{32}^{ \pm(q+1)}, \chi_{2}^{ \pm(q+1)}$. The mass matrix extracted from (A.5) yields four massless fields, identified with the Goldstone bosons of the new gauge bosons $Y_{L, R}^{ \pm(q+1)}$, and defined by

$$
\begin{aligned}
G_{Y_{L}}^{ \pm(q+1)}= & \frac{1}{\sqrt{u^{\prime 2} w^{2} w^{\prime 4}+\left(u^{\prime 2}-w^{2}\right)^{4}+\left(u^{\prime 6}-3 u^{\prime 2} w^{4}+2 w^{6}\right) w^{\prime 2}+w^{4} w^{\prime 4}}} \times \\
& \left\{\left[-\left(u^{\prime 2}-w^{2}\right)^{2}-w^{2} w^{\prime 2}\right] \phi_{23}^{ \pm(q+1)}+u^{\prime} w w^{\prime 2} \phi_{32}^{ \pm(q+1)}+u^{\prime} w^{\prime}\left(u^{\prime 2}-w^{2}\right) \chi_{2}^{ \pm(q+1)}\right\} \\
G_{Y_{R}}^{ \pm(q+1)}= & \frac{1}{\sqrt{\left(u^{\prime 2}-w^{2}\right)^{2}+w^{2} w^{\prime 2}}}\left\{\left(w^{2}-u^{\prime 2}\right) \phi_{32}^{ \pm(q+1)}+w w^{\prime} \chi_{2}^{ \pm(q+1)}\right\} .
\end{aligned}
$$

The other physical fields are massive with corresponding masses,

$$
\begin{aligned}
\mathcal{H}_{Y}^{ \pm(q+1)} & =\frac{1}{\sqrt{w^{\prime 2}\left(w^{2}+u^{\prime 2}\right)+\left(u^{\prime 2}-w^{2}\right)^{2}}}\left\{w^{\prime} u^{\prime} \phi_{23}^{ \pm(q+1)}+w w^{\prime} \phi_{32}^{ \pm(q+1)}+\left(u^{\prime 2}-w^{2}\right) \chi_{2}^{ \pm(q+1)}\right\} \\
m_{\mathcal{H}_{Y}}^{2} & =\frac{\zeta_{6}}{2\left(u^{\prime 2}-w^{2}\right)}\left\{u^{\prime 4}+u^{\prime 2}\left(w^{\prime 2}-2 w^{2}\right)+w^{2}\left(w^{2}+w^{\prime 2}\right)\right\}
\end{aligned}
$$

For $(q-1)$ and $2 q$-charged scalars, $\sigma_{23}^{ \pm(q-1)}$ and $\sigma_{33}^{ \pm 2 q}$ are already physical fields, with masses

$$
\begin{aligned}
& m_{\sigma_{23}}^{2}=\frac{1}{4}\left\{\zeta_{5} w^{\prime 2}-4 \kappa_{2} \Lambda^{2}+\frac{\left(u^{2}-u^{\prime 2}\right)\left(2 u^{2}-u^{\prime 2}-w^{2}\right)\left[2 \lambda_{2}\left(u^{2}-w^{2}\right)\left(u^{\prime 2}-w^{2}\right)+\zeta_{6} w^{2} w^{\prime 2}\right]}{u^{2} \Lambda^{2}\left(u^{\prime 2}-w^{2}\right)}\right\} \\
& m_{\sigma_{33}}^{2}=\frac{1}{2}\left\{\zeta_{5} w^{\prime 2}-2 \kappa_{2} \Lambda^{2}+\frac{\left(u^{2}-u^{\prime 2}\right)\left(u^{2}-w^{2}\right)\left[2 \lambda_{2}\left(u^{2}-w^{2}\right)\left(u^{\prime 2}-w^{2}\right)+\zeta_{6} w^{2} w^{\prime 2}\right]}{u^{2}\left(u^{\prime 2}-w^{2}\right) \Lambda^{2}}\right\} .
\end{aligned}
$$

\subsection{Gauge-boson sector}

Let us now study the physical gauge boson states and their masses. In the non-Hermitian gauge boson sector, there are three kinds of left-right gauge bosons, $W_{L, R}^{ \pm}, X_{L, R}^{ \pm q}, Y_{L, R}^{ \pm(q+1)}$. The fields $W_{L, R}^{ \pm}$, which are defined as $W_{L}^{ \pm}=\frac{1}{\sqrt{2}}\left(A_{1 L} \mp i A_{2 L}\right)$ and $W_{R}^{ \pm}=\frac{1}{\sqrt{2}}\left(A_{1 R} \mp i A_{2 R}\right)$, mix through the mass matrix,

$$
\frac{g_{L}^{2}}{4}\left(\begin{array}{lc}
u^{2}+u^{\prime 2} & -2 t_{R} u u^{\prime} \\
-2 t_{R} u u^{\prime} & t_{R}^{2}\left(u^{2}+u^{\prime 2}+2 \Lambda^{2}\right)
\end{array}\right) .
$$

Diagonalizing this matrix, the eigenstates and masses are given by

$$
\begin{aligned}
& W_{1}=c_{\xi} W_{L}-s_{\xi} W_{R}, \quad m_{W_{1}}^{2} \simeq \frac{g_{L}^{2}}{4}\left\{u^{2}+u^{\prime 2}-\frac{4 t_{R}^{2} u^{2} u^{\prime 2}}{\left(t_{R}^{2}-1\right)\left(u^{2}+u^{\prime 2}\right)+2 t_{R}^{2} \Lambda^{2}}\right\}, \\
& W_{2}=s_{\xi} W_{L}+c_{\xi} W_{R}, \quad m_{W_{2}}^{2} \simeq \frac{g_{R}^{2}}{4}\left\{u^{2}+u^{\prime 2}+2 \Lambda^{2}+\frac{4 t_{R}^{2} u^{2} u^{\prime 2}}{\left(t_{R}^{2}-1\right)\left(u^{2}+u^{\prime 2}\right)+2 t_{R}^{2} \Lambda^{2}}\right\},
\end{aligned}
$$

where $\Lambda \gg u, u^{\prime}$ and $t_{2 \xi}=\frac{-4 t_{R} u u^{\prime}}{2 t_{R}^{2} \Lambda^{2}+\left(t_{R}^{2}-1\right)\left(u^{2}+u^{\prime 2}\right)}$ and $t_{R}=\frac{g_{R}}{g_{L}}$. $W_{1}$ is identified as the SM $W$ boson, which implies $u^{2}+u^{\prime 2} \simeq(246 \mathrm{GeV})^{2}$. $W_{2}$ is a physical heavy state, with mass at the new physics scale. 
The mass matrix of the fields $X_{L}^{ \pm q}=\frac{1}{\sqrt{2}}\left(A_{4 L} \mp i A_{5 L}\right)$ and $X_{R}^{ \pm q}=\frac{1}{\sqrt{2}}\left(A_{4 R} \mp i A_{5 R}\right)$ is

$$
\frac{g_{L}^{2}}{4}\left(\begin{array}{lc}
u^{2}+w^{2} & -2 t_{R} u w \\
-2 t_{R} u w & t_{R}^{2}\left(u^{2}+w^{\prime 2}+w^{2}+2 \Lambda^{2}\right)
\end{array}\right),
$$

and yields two physical heavy states with masses

$$
\begin{aligned}
& X_{1}^{ \pm q} \simeq c_{\xi_{1}} X_{L}^{ \pm}-s_{\xi_{1}} X_{R}^{ \pm q} \\
& m_{X_{1}}^{2} \simeq \frac{g_{L}^{2}}{4}\left\{u^{2}+w^{2}+\frac{4 t_{R}^{2} u^{2} w^{2}}{u^{2}+w^{2}-t_{R}^{2}\left(u^{2}+w^{\prime 2}+w^{2}+2 \Lambda^{2}\right)}\right\} \\
& X_{2}^{ \pm q} \simeq s_{\xi_{1}} X_{L}^{ \pm}+c_{\xi_{1}} X_{R}^{ \pm q} \\
& m_{X_{2}}^{2} \simeq \frac{g_{R}^{2}}{4}\left\{u^{2}+w^{2}+w^{\prime 2}+2 \Lambda^{2}-\frac{4 u^{2} w^{2}}{u^{2}+w^{2}-t_{R}^{2}\left(u^{2}+w^{\prime 2}+w^{2}+2 \Lambda^{2}\right)}\right\}
\end{aligned}
$$

with $t_{2 \xi_{1}}=\frac{4 t_{R} u w}{u^{2}+w^{2}-t_{R}^{2}\left(u^{2}+w^{2}+w^{2}+2 \Lambda^{2}\right)}$.

The fields, $Y_{L}^{ \pm(1+q)}=\frac{1}{\sqrt{2}}\left(A_{6 L} \pm i A_{7 L}\right)$ and $Y_{R}^{ \pm(1+q)}=\frac{1}{\sqrt{2}}\left(A_{6 R} \pm i A_{7 R}\right)$, have the following mass matrix

$$
\frac{g_{L}^{2}}{4}\left(\begin{array}{cc}
u^{\prime 2}+w^{2} & -2 t_{R} u^{\prime} w \\
-2 t_{R} u^{\prime} w & t_{R}^{2}\left(u^{\prime 2}+w^{\prime 2}+w^{2}\right)
\end{array}\right),
$$

which provides physical heavy states with masses

$$
\begin{aligned}
Y_{1}^{ \pm(1+q)} & =c_{\xi_{2}} Y_{L}^{ \pm(1+q)}-s_{\xi_{2}} Y_{R}^{ \pm(1+q)}, \\
m_{Y_{1}}^{2} & \simeq \frac{g_{L}^{2}}{4}\left\{u^{\prime 2}+w^{2}+\frac{4 t_{R}^{2} u^{\prime 2} w^{2}}{u^{\prime 2}+w^{2}-t_{R}^{2}\left(u^{\prime 2}+w^{\prime 2}+w^{2}\right)}\right\}, \\
Y_{2}^{ \pm(1+q)} & =s_{\xi_{2}} Y_{L}^{ \pm(1+q)}+c_{\xi_{2}} Y_{R}^{ \pm(1+q)}, \\
m_{Y_{2}}^{2} & \simeq \frac{g_{R}^{2}}{4}\left\{u^{\prime 2}+w^{\prime 2}+w^{2}-\frac{4 u^{\prime 2} w^{2}}{u^{\prime 2}+w^{2}-t_{R}^{2}\left(u^{\prime 2}+w^{\prime 2}+w^{2}\right)}\right\},
\end{aligned}
$$

where the mixing angle $\xi_{2}$ satisfies $t_{2 \xi_{2}}=\frac{4 t_{R} u^{\prime} w}{u^{\prime 2}+w^{2}-t_{R}^{2}\left(u^{\prime 2}+w^{\prime 2}+w^{2}\right)}$.

The neutral gauge bosons, $A_{3 L}, A_{3 R}, A_{8 L}, A_{8 R}, B$, mix via a $5 \times 5$ mass matrix. In order to find its eigenstates, we first work with a new basis

$$
\begin{aligned}
A & =s_{W} A_{3 L}+c_{W}\left\{\frac{t_{W}}{t_{R}} A_{3 R}+\beta t_{W} A_{8 L}+\beta \frac{t_{W}}{t_{R}} A_{8 R}+\frac{t_{W}}{t_{X}} B\right\}, \\
Z_{L} & =c_{W} A_{3 L}-s_{W}\left\{\frac{t_{W}}{t_{R}} A_{3 R}+\beta t_{W} A_{8 L}+\beta \frac{t_{W}}{t_{R}} A_{8 R}+\frac{t_{W}}{t_{X}} B\right\}, \\
Z_{L}^{\prime} & =\varsigma_{1} t_{X} t_{W} \beta A_{3 R}-\frac{t_{W}}{\varsigma_{1} t_{X} t_{R}} A_{8 L}+\varsigma_{1} t_{X} t_{W} \beta^{2} A_{8 R}+\varsigma_{1} t_{R} t_{W} \beta B, \\
Z_{R} & =-\frac{\varsigma_{1}}{\varsigma} A_{3 R}+\varsigma_{1} t_{X}^{2} \beta A_{8 R}+\varsigma_{1} t_{X} t_{R} B, \\
Z_{R}^{\prime} & =\varsigma\left(t_{R} A_{8 R}-t_{X} \beta B\right),
\end{aligned}
$$

where $t_{X}=\frac{g_{X}}{g_{L}}, \varsigma=\frac{1}{\sqrt{t_{R}^{2}+\beta^{2} t_{X}^{2}}}, \varsigma_{1}=\frac{1}{\sqrt{t_{R}^{2}+\left(1+\beta^{2}\right) t_{X}^{2}}}$, and $s_{W}=\frac{t_{X} t_{R}}{\sqrt{t_{X}^{2}\left(1+\beta^{2}\right)+t_{R}^{2}\left(1+t_{X}^{2}\left(1+\beta^{2}\right)\right)}}$. 
The gauge boson $A$ is massless and decouples, therefore it is identified with the photon field. The remaining fields, $Z_{L}, Z_{L}^{\prime}, Z_{R}, Z_{R}^{\prime}$, mix among themselves through a $4 \times 4$ mass matrix. Given that $w, \Lambda \gg u, u^{\prime}$, the mass matrix elements that connect $Z_{L}$ to $Z_{L}^{\prime}, Z_{R}, Z_{R}^{\prime}$ are very suppressed. The mass matrix can be diagonalized using the seesaw formula to separate the light state $Z_{L}$ from the heavy ones $Z_{L}^{\prime}, Z_{R}, Z_{R}^{\prime}$. Thus, the SM $Z$ boson is identified with $Z_{L}$ whose mass is $m_{Z}^{2} \simeq \frac{g_{L}^{2}}{4 c_{W}^{2}}\left(u^{2}+u^{\prime 2}\right)$. For the heavy neutral gauge bosons, the mass matrix elements are proportional to the square of the $w, w^{\prime}, \Lambda$ energy scales. In the general case, it is very difficult to find the physical heavy states. However, if there is a hierarchy between two energy scales $w, w^{\prime}$ and $\Lambda$, we can find them. In particular, in the limit $\Lambda \gg w, w^{\prime}$, the physical heavy states are

$$
\begin{aligned}
\mathcal{Z}_{L}^{\prime} & \simeq Z_{L}^{\prime}, \quad m_{\mathcal{Z}_{L}^{\prime}}^{2} \simeq \frac{g_{L}^{2}}{3} \frac{\left(1+\varsigma_{1}^{2} t_{R}^{2} t_{X}^{2} \beta^{2}\right)^{2} t_{W}^{2} w^{2}}{\varsigma_{1}^{2} t_{R}^{2} t_{X}^{2}} \\
\mathcal{Z}_{R} & \simeq c_{\xi_{3}} Z_{R}-s_{\xi_{3}} Z_{R}^{\prime}, \quad \mathcal{Z}_{R}^{\prime} \simeq s_{\xi_{3}} Z_{R}+c_{\xi_{3}} Z_{R}^{\prime} \\
m_{\mathcal{Z}_{R}}^{2} & \simeq \frac{g_{L}^{2}}{3} \frac{3 w^{\prime 2}\left[t_{R}^{2}+t_{X}^{2}\left(1+\beta^{2}\right)\right]^{2}+w^{2}\left[3 t_{R}^{4}+2 t_{R}^{2} t_{X}^{2}(3+\sqrt{3} \beta)+t_{X}^{4}\left(3+2 \sqrt{3} \beta+\beta^{2}\right)\right]}{\varsigma_{1}^{-2}\left[4+\left(3+2 \sqrt{3} \beta+\beta^{2}\right)\left(t_{X}^{2} / t_{R}^{2}\right)\right]} \\
m_{\mathcal{Z}_{\mathcal{R}}^{\prime}}^{2} & \simeq \frac{g_{L}^{2}}{3}\left\{4 t_{R}^{2}+t_{X}^{2}\left(3+2 \sqrt{3} \beta+\beta^{2}\right)\right\} \Lambda^{2}
\end{aligned}
$$

where the $Z_{R^{-}} Z_{R}^{\prime}$ mixing angle is

$$
t_{2 \xi_{3}}=\frac{2 t_{R}\left[\sqrt{3} t_{R}^{2}+\beta(3+\sqrt{3} \beta) t_{X}^{2}\right] \sqrt{t_{R}^{2}+t_{X}^{2}\left(1+\beta^{2}\right)}}{2 t_{R}^{4}+t_{R}^{2} t_{X}^{2}\left(3-2 \sqrt{3} \beta+\beta^{2}\right)-\beta^{2}\left(3+2 \sqrt{3} \beta+\beta^{2}\right) t_{X}^{4}} .
$$

With the physical states properly identified, we list in appendix B the most important interactions between the gauge bosons and fermions in the model. Now we turn to thed to discussion of the dark matter phenomenology.

\section{Dark matter}

Despite the multitude of evidence for the existence of dark matter in our universe, its nature remains a mystery and it is one of the most exciting and important open questions in basic science [2]. In this work, we will investigate the possible dark matter candidates in our model and discuss the relevant observables, namely relic density and direct detection cross section. Indirect detection is not very relevant in our model because we will be discussing multi-TeV scale dark matter, a regime for which indirect dark matter detection cannot probe the thermal annihilation cross section [59]. In this section we will briefly address the possibility of scalar and fermion WIMP dark matter. First we note that, in some limiting cases, it is very similar to the one present in $3-3-1$ models $[43,44,47,51,60,61]$. As a result we opt for a more sketcky presentation here, primarily aimed at establishing the viabiblity of the present scenario, rather than covering it in full generality. Our main goal in this section is to illustrate our reasoning concerning the origin of the dark matter stability in a unified framework involving 3-3-1 and left-right symmetries, by simply showing that we have do viable dark matter candidates. 
That said, we have seen that the $W$-parity symmetry is exact and unbroken by the VEVs. Thus, the lightest neutral $W$-particle is stable and can be potentially responsible for the observed DM relic density. For concreteness we will study the model with $q=0$, i.e. $\beta=-\frac{1}{\sqrt{3}}$. The neutral $W$-particles include a fermion $N_{a}^{0}$, a vector gauge boson $X_{1,2}^{0}$, and a scalar $\mathcal{H}_{1,2}^{0} \cdot{ }^{4}$

\subsection{Scalar dark matter}

\subsubsection{Relic density}

Suppose that $\mathcal{H}_{2}^{0}$ is the lightest $W$-particle (LWP). It cannot decay and can only be produced in pairs. The scalar dark matter has only $s$-wave contribution to the annihilation cross-section. Hence, the dark matter abundance can be approximated as

$$
\Omega_{\mathcal{H}_{2}} h^{2} \simeq \frac{0.1 \mathrm{pb}}{\left\langle\sigma v_{\text {rel }}\right\rangle},
$$

where $\left\langle\sigma v_{\text {rel }}\right\rangle$ is the thermally averaged cross-section times relative velocity. As our candidates are naturally heavy at the new physics scale, the SM Higgs portal is inaccessible. The main contribution to the cross-section times relative velocity is determined by the direct annihilation channel $\mathcal{H}_{2}^{0 *} \mathcal{H}_{2}^{0} \rightarrow H_{1} H_{1}$ or mediated by new scalars. In the limit $\Lambda \gg w, w^{\prime} \gg u, u^{\prime}$, the interaction between $\mathcal{H}_{2}^{0}$ and $H_{1}$ is approximated as

$$
\mathcal{L}_{\mathcal{H}_{2}^{0}-H_{1}}=\frac{\left[\lambda_{2} u^{2}+\lambda_{1}\left(u^{2}+u^{\prime 2}\right)\right] c_{\theta_{q}}^{2}}{u^{2}+u^{\prime 2}} \mathcal{H}_{2}^{0} \mathcal{H}_{2}^{0} H_{1} H_{1}
$$

It can be shown that the new Higgs portal gives a contribution of the same magnitude as the one above. Therefore in our estimate it is enough to consider only the $\mathcal{H}_{2}^{0 *} \mathcal{H}_{2}^{0} \rightarrow H_{1} H_{1}$ contact interaction. The average cross-section times relative velocity is

$$
\left\langle\sigma v_{\text {rel }}\right\rangle=\frac{1}{16 \pi m_{\mathcal{H}_{2}}^{2}}\left\{\frac{\left[\lambda_{2} u^{2}+\lambda_{1}\left(u^{2}+u^{\prime 2}\right)\right] c_{\theta_{q}}^{2}}{u^{2}+u^{\prime 2}}\right\}^{2}\left(1-\frac{\left\langle v^{2}\right\rangle}{2}-\frac{m_{H_{1}}^{2}}{m_{\mathcal{H}_{2}}^{2}}\right),
$$

where the dark matter velocity $v$ satisfies $\left\langle v^{2}\right\rangle=\frac{3}{2 x_{F}}$, with $x_{F}=m_{\mathcal{H}_{2}} / T_{F} \sim 20$ at the freezeout temperature [1]. Since $m_{\mathcal{H}_{2}}^{2} \gg m_{H_{1}}^{2}$, we approximate

$$
\left\langle\sigma v_{\text {rel }}\right\rangle=\left\{\frac{\alpha}{150 \mathrm{GeV}}\right\}^{2}\left\{\frac{\left[\lambda_{2} u^{2}+\lambda_{1}\left(u^{2}+u^{\prime 2}\right)\right] c_{\theta_{q}}^{2}}{u^{2}+u^{\prime 2}}\right\}^{2}\left\{\frac{2.656 \mathrm{TeV}}{m_{\mathcal{H}_{2}}}\right\}^{2} .
$$

Thus, the dark matter candidate $\mathcal{H}_{2}^{0}$ reproduces the correct relic density, $\Omega_{\mathcal{H}_{2}} h^{2} \simeq 0.11$ [58], if $\left\langle\sigma v_{\text {rel }}\right\rangle \simeq 1 \mathrm{pb}$, or

$$
m_{\mathcal{H}_{2}} \simeq \frac{\left[\lambda_{2} u^{2}+\lambda_{1}\left(u^{2}+u^{\prime 2}\right)\right] c_{\theta_{q}}^{2}}{u^{2}+u^{\prime 2}} \times 2.656 \mathrm{TeV} \sim 2.5 \mathrm{TeV}
$$

for scalar couplings of $\mathcal{O}(1)$, and using the fact that $\alpha^{2} /(150 \mathrm{GeV})^{2} \simeq 1 \mathrm{pb}$. Furthermore, the above condition implies

$$
m_{\mathcal{H}_{2}}<2.66\left(\lambda_{1}+\lambda_{2}\right) \mathrm{TeV}<67 \mathrm{TeV},
$$

\footnotetext{
${ }^{4}$ The other two dark matter models with $q= \pm 1$ can be examined in a similar way.
} 
where the upper limit comes from the perturbativity bound $\lambda_{1}, \lambda_{2}<4 \pi$. Therefore, the dark matter mass may be in the range few $\mathrm{TeVs}$ to $67 \mathrm{TeV}$, depending on its interaction strength with the SM Higgs boson.

\subsubsection{Direct detection}

The detection through low energy nuclear recoils constitute a clear signature for dark matter particles. Since no signal has been observed thus far, stringent limits have been derived on the dark matter-nucleon scattering cross section [62-70].

In the scalar dark matter scenario, this scattering takes place through the t-channel exchange of a $\mathcal{Z}_{L}^{\prime}$ and a heavy scalar $\mathcal{H}_{0}^{1}$. This scenario is similar to the one studied in [50], where it has been shown that one can obey direct detection limits from the XENON1T experiment with 2 years of data for the dark matter masses above $3 \mathrm{TeV}$, while reproducing the correct relic density. We emphasize that our goal here was simply to show that we do have viable dark matter candidates, rather than investigating in detail their phenomenology. In fact, the latter is substantially more complex than found in previous 3-3-1 models. However it suffices to exemplify the viability of our dark matter candidates under certain assumptions.

\subsection{Fermion dark matter}

\subsubsection{Relic density}

Let us now assume that the LWP is one of the neutral fermions denoted by $N$. The model predicts that $N$ is a Dirac fermion. The covariant derivative (i.e., gauge interactions) dictates the dark matter phenomenology. The dark matter might annihilate into SM particles via the well known $Z^{\prime}$ portal with predictive observables [53, 71]. The relic density is governed by s-channel annihilations into SM fermions, whose interactions are presented in appendix B. Assuming that the mixing between the gauge boson $\mathcal{Z}_{L}^{\prime}$ and the other gauge bosons to be small, which can be achived by taking $\Lambda \gg w, w^{\prime} \gg u, u^{\prime}$, one finds the relic density to be achieved either by annihilation into fermion pairs, or into $\mathcal{Z}_{L}^{\prime} \mathcal{Z}_{L}^{\prime}$. The role of $\mathcal{Z}_{R}$ is analogous to $Z_{N}$ in the 3-3-1-1 model [51], which is not discussed further. In figure 1 we show the relic density curve in green.

\subsubsection{Direct detection}

The dark matter-nucleon scattering is mostly driven by the t-channel exchange of the $\mathcal{Z}_{L}^{\prime}$ gauge boson. This scattering is very efficient since it is governed simply the couplings with up and down quarks without much freedom. Taking into account the current and projected sensitivities on the dark matter-nucleon scattering cross-section, one can conclude that the dark matter mass must lie in the few TeV scale, as already investigated in [61]. Notice that this conclusion holds for a Dirac fermion (the possibility of having a Majorana fermion has already been ruled out by direct detection data [61]). The Majorana dark matter case leads to an annihilation rate which is helicity suppressed and therefore the range of parameter space that yields the correct relic density is smaller compared to the Dirac fermion scenario, only $\mathcal{Z}_{L}^{\prime}$ masses up to $2.5 \mathrm{TeV}$ can reproduce the correct relic density in the $\mathcal{Z}_{L}^{\prime}$ resonance regime. 


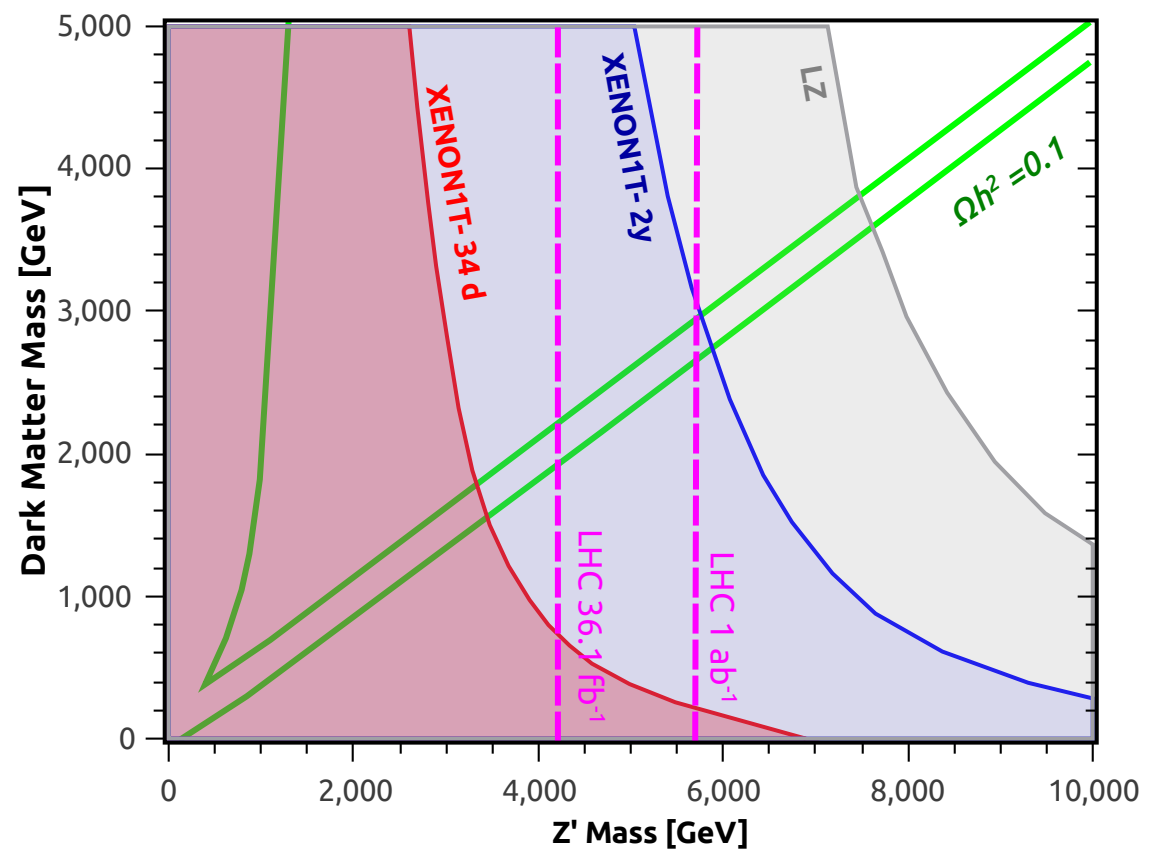

Figure 1. Summary plot for the fermion dark matter. Relic density curve (green), LHC (pink) and current direct detection limit from XENON1T-34 days (red) [67], projected from XENON1T-2 years (blue) [74] and LZ (gray) [75] are overlaid. See text for detail.

\subsubsection{Collider}

As for collider bounds, LHC results based on heavy dilepton resonance searches with $13.3 \mathrm{fb}^{-1}$ of integrated luminosity exclude $\mathcal{Z}_{L}^{\prime}$ masses below few $3.8 \mathrm{TeV}$ [61]. This is very important because in light of this bound, the Majorana dark matter case has already been ruled out, since the entire parameter space which yields the correct relic density in within the LHC exclusion region.

In light of the importance of this collider bound we took the opportunity to do a rescalling with the luminosity to obtain current and projected limits on the $\mathcal{Z}_{L}^{\prime}$ mass in our model for $36.1 \mathrm{fb}^{-1}$ and $1000 \mathrm{fb}^{-1}$ keeping the center-of-energy of $13 \mathrm{TeV}$, using the collider reach tool introduced in. ${ }^{5}$ The limits read $m_{\mathcal{Z}_{L}^{\prime}}>4.2 \mathrm{TeV}$ and $m_{\mathcal{Z}_{L}^{\prime}}>5.7 \mathrm{TeV}$, respectively. These bounds can be seen as vertical lines in figure 1 . We emphasize that other limits stemming from electroweak precision or low energy physics are subdominant thus left out of the discussion $[72,73]$. In summary, one can conclude that our model can successfully accommodate a Dirac fermion dark matter in agreement with existing and projected limits near the $\mathcal{Z}_{L}^{\prime}$ resonance. Once again, our discussion clearly shows the present of a viable dark matter candidate. We stress that, in full generality, the phenomenology of our model is substantially more complex than presented in previous literature. Here we only consider simple benchmarks, for which a detailed study of the dark matter phenomenology has

\footnotetext{
${ }^{5}$ http: $/ /$ collider-reach.web.cern.ch/?rts1 $=13 \&$ lumi $1=3.2 \&$ rts $2=13 \&$ lumi2 $=13.3 \&$ pdf $=$ MSTW 2008 nnlo68cl.LHgrid.
} 
already been performed earlier, hence we have not repeated it here. A complete study of the phenomenological implications of our model lies beyond the scope of this paper and will be taken up elsewhere. Nevertheless, our results suffice to establish the consistency of our dark matter candidates.

\subsection{Gauge-boson dark matter}

\subsubsection{Relic density}

Finally, let us give a comment on the possibility of vector gauge boson dark matter. In this case one assumes that the LWP is the gauge boson $X_{1}^{0}$. It can annihilate into SM particles via following channels,

$$
X_{1}^{0} X_{1}^{0 *} \rightarrow W_{1}^{+} W_{1}^{-}, Z_{L} Z_{L}, H_{1} H_{1}, \nu \nu^{c}, l l^{c}, q q^{c},
$$

where $\nu=\nu_{e}, \nu_{\mu}, \nu_{\tau}, l=e, \mu, \tau, q=u, d, c, s, t, b$. However, the dominant channels are $X_{1}^{0} X_{1}^{0 *} \rightarrow W_{1}^{+} W_{1}^{-}, Z_{L} Z_{L}$. Our predicted result is similar to the one given in [45]. The dark matter relic abundance is approximately given as

$$
\Omega_{X_{1}} h^{2} \simeq 10^{-3} \frac{m_{W}^{2}}{m_{X_{1}}^{2}}
$$

Since the annihilation cross section is large and it is dictated by gauge interactions, the abundance of this vector dark matter is too small. In the context of thermal dark matter production, the vector dark matter in our model can contribute to only a tiny fraction of the dark matter abundance in our universe. A similar conclusion has been found in [43].

One way to circumvent the vector dark matter underabundance is by abdicating thermal production and tie its abundance to inflation, where the inflaton decay or the gravitational mechanism would generate the correct dark matter abundance [55]. Alternatively, we mention that vector DM could be just part of the overall cosmological dark matter within a multicomponent thermal dark matter scenario.

\section{Conclusions}

We have proposed a model of flipped trinification that encompasses the nice features of left-right and 3-3-1 models, while providing an elegant explanation for the origin of matter parity and dark matter stability, which follows as a remnant of the gauge symmetry. The model offers a natural framework for three types of dark matter particles, which is an uncommon feature in UV complete models. One can have a Dirac fermion, as well as a scalar dark matter particle, with masses at the few $\mathrm{TeV}$ scale. Both scenarios reproduce the correct relic density, while satisfying existing limits, in the context of thermal freeze-out. As for the vector case, thermal production leads to an under-abundant dark matter. We have also discussed other features of the model such as the symmetry breaking, driven by a minimal scalar content, but sufficient to account for realistic fermion masses. In summary, we have presented a viable theory of flipped trinification able to account naturally for the origin of matter parity and dark matter. 


\section{Acknowledgments}

The authors thank Carlos Yaguna, Carlos Pires, Alex Dias. This research is funded by Vietnam National Foundation for Science and Technology Development (NAFOSTED) under grant numbers 103.01-2016.77 and 103.01-2017.05, and is supported by the Spanish grants FPA2014-58183-P and SEV-2014-0398 (MINECO), and PROMETEOII/2014/084 (Generalitat Valenciana). FSQ acknowledges support from MEC, UFRN and ICTP-SAIFR FAPESP grant 2016/01343-7. C.A.V-A. acknowledges financial support from Cátedras CONACYT, proyect 749 .

\section{A Relevant scalar mass terms}

The scalar fields mix according to the class they belong, and their relevant corresponding mass terms are derived as

$$
\begin{aligned}
& V_{\text {mass }}^{A}=A_{2}^{2}\left\{\frac{w^{2} u^{\prime 2}\left(2 \lambda_{2}\left(w^{2}-u^{\prime 2}\right)+\zeta_{6} w^{\prime 2}\right)}{4 u^{2}\left(u^{\prime 2}-w^{2}\right)}\right\}+A_{2} A_{3}\left\{\frac{w^{2} u^{\prime}\left(2 \lambda_{2}\left(w^{2}-u^{\prime 2}\right)+\zeta_{6} w^{\prime 2}\right)}{2 u\left(u^{\prime 2}-w^{2}\right)}\right\} \\
& +A_{2} A_{4}\left\{\frac{w u^{\prime 2}\left(2 \lambda_{2}\left(w^{2}-u^{\prime 2}\right)+\zeta_{6} w^{\prime 2}\right)}{2 u\left(u^{\prime 2}-w^{2}\right)}\right\}+A_{3}^{2}\left\{\frac{w^{2}\left(2 \lambda_{2}\left(w^{2}-u^{\prime 2}\right)+\zeta_{6} w^{\prime 2}\right)}{4\left(u^{\prime 2}-w^{2}\right)}\right\} \\
& +A_{3} A_{4}\left\{\frac{w u^{\prime}\left(2 \lambda_{2}\left(w^{2}-u^{\prime 2}\right)+\zeta_{6} w^{\prime 2}\right)}{2\left(u^{\prime 2}-w^{2}\right)}\right\}+A_{4}^{2}\left\{\frac{u^{\prime 2}\left(2 \lambda_{2}\left(w^{2}-u^{\prime 2}\right)+\zeta_{6} w^{\prime 2}\right)}{4\left(u^{\prime 2}-w^{2}\right)}\right\} \text {. } \\
& V_{\text {mass }}^{S}=\left(\kappa_{1}+\kappa_{2}\right) \Lambda^{2} S_{1}^{2}+\left\{\frac{\left(u^{\prime 2}-u^{2}\right)\left[2 \lambda_{2}\left(u^{2}-w^{2}\right)\left(u^{\prime 2}-w^{2}\right)+\zeta_{6} w^{2} w^{\prime 2}\right]}{u\left(u^{\prime 2}-w^{2}\right) \Lambda}+\zeta_{2} u \Lambda\right\} S_{1} S_{2}+\zeta_{2} u^{\prime} \Lambda S_{1} S_{3} \\
& +\zeta_{2} w \Lambda S_{1} S_{4}+\zeta_{4} w^{\prime} \Lambda S_{1} S_{5}+\frac{2\left(u^{\prime 2}-w^{2}\right)\left[2\left(\lambda_{1}+\lambda_{2}\right) u^{4}-\lambda_{2} u^{\prime 2} w^{2}\right]+\zeta_{6} u^{\prime 2} w^{2} w^{\prime 2}}{4 u^{2}\left(u^{\prime 2}-w^{2}\right)} S_{2}^{2} \\
& +\frac{u^{\prime}\left[4 \lambda_{1} u^{2}+w^{2}\left(2 \lambda_{2}+\frac{\zeta_{6} w^{\prime 2}}{w^{2}-u^{\prime 2}}\right)\right]}{2 u} S_{2} S_{3}+\frac{w\left(4 \lambda_{1} u^{2}+u^{\prime 2}\left[2 \lambda_{2}+\frac{\zeta_{6} w^{\prime 2}}{w^{2}-u^{\prime 2}}\right]\right)}{2 u} S_{2} S_{4}+\zeta_{1} u w^{\prime} S_{2} S_{5} \\
& +\left\{\lambda_{1} u^{\prime 2}+\lambda_{2}\left(u^{\prime 2}-\frac{w^{2}}{2}\right)-\frac{\zeta_{6} w^{2} w^{\prime 2}}{4\left(w^{2}-u^{\prime 2}\right)}\right\} S_{3}^{2}+\frac{w u^{\prime}}{2}\left\{4 \lambda_{1}+2 \lambda_{2}+\frac{\zeta_{6} w^{\prime 2}}{w^{2}-u^{\prime 2}}\right\} S_{3} S_{4}+\zeta_{1} u^{\prime} w^{\prime} S_{3} S_{5} \\
& +\left\{\lambda_{1} w^{2}+\lambda_{2}\left(w^{2}-\frac{u^{\prime 2}}{2}\right)-\frac{\zeta_{6} u^{\prime 2} w^{\prime 2}}{4\left(w^{2}-u^{\prime 2}\right)}\right\} S_{4}^{2}+w w^{\prime}\left(\zeta_{1}+\zeta_{6}\right) S_{4} S_{5}+\lambda w^{\prime 2} S_{5}^{2} . \\
& V_{\text {mass }}^{\text {singly-charged }}=\frac{\left(u^{2}-u^{\prime 2}\right)^{2}\left[2 \lambda_{2}\left(u^{\prime 2}-w^{2}\right)\left(u^{\prime 2}-w^{2}\right)+\zeta_{6} w^{2} w^{\prime 2}\right]}{4 u^{2}\left(u^{\prime 2}-w^{2}\right) \Lambda^{2}} \sigma_{12}^{-} \sigma_{12}^{+} \\
& +\left\{\lambda_{2}\left(u^{2}-w^{2}\right)+\frac{\zeta_{6} w^{2} w^{\prime 2}}{2\left(u^{\prime 2}-w^{2}\right)}\right\} \phi_{12}^{-} \phi_{12}^{+}+\left\{\frac{\lambda_{2} u^{\prime 2}\left(u^{2}-w^{2}\right)}{u^{2}}+\frac{\zeta_{6} w^{2} w^{\prime 2} u^{\prime 2}}{2 u^{2}\left(u^{\prime 2}-w^{2}\right)}\right\} \phi_{21}^{+} \phi_{21}^{-} \\
& +\left\{\frac{\left(u^{\prime 2}-u^{2}\right)\left(2 \lambda_{2}\left(u^{2}-w^{2}\right)\left(u^{\prime 2}-w^{2}\right)+\zeta_{6} w^{2} w^{\prime 2}\right.}{2 \sqrt{2} u\left(u^{\prime 2}-w^{2}\right) \Lambda}\left(\sigma_{12}^{-} \phi_{12}^{+}+\frac{u^{\prime}}{u} \sigma_{12}^{-} \phi_{21}^{+}\right)\right. \\
& \left.+\left(\frac{\lambda_{2} u^{\prime 2}\left(u^{2}-w^{2}\right)}{u^{2}}+\frac{\zeta_{6} u^{\prime 2} w^{2} w^{\prime 2}}{2 u^{2}\left(u^{\prime 2}-w^{2}\right)}\right) \phi_{12}^{-} \phi_{21}^{+}+H . c\right\} \text {. }
\end{aligned}
$$




$$
\begin{aligned}
V_{\text {mass }}^{q-\text { charged }}= & \left\{\lambda_{2}\left(u^{2}-u^{\prime 2}\right)+\frac{\zeta_{6} u^{\prime 2} w^{\prime 2}}{2\left(u^{\prime 2}-w^{2}\right)}\right\} \phi_{13}^{+q} \phi_{13}^{-q}+\left\{\frac{\lambda_{2} w^{2}\left(u^{2}-u^{\prime 2}\right)}{u^{2}}+\frac{\zeta_{6} u^{\prime 2} w^{\prime 2} w^{2}}{2 u^{2}\left(u^{\prime 2}-w^{2}\right)}\right\} \phi_{31}^{+q} \phi_{31}^{-q} \\
& +\left\{\frac{\left(u^{2}-u^{\prime 2}\right)\left(u^{2}-w^{2}\right)\left(2 \lambda\left(u^{2}-w^{2}\right)\left(u^{\prime 2}-w^{2}\right)+\zeta_{6} w^{2} w^{\prime}+\zeta_{5}\left(u^{\prime 2}-w^{2}\right) u^{2} w^{\prime 2} \Lambda^{2}\right)}{4 u^{2} \Lambda^{2}\left(u^{\prime 2}-w^{2}\right)}\right\} \sigma_{13}^{+q} \sigma_{13}^{-q} \\
& +\frac{1}{2}\left\{\zeta_{6}\left(u^{2}-w^{2}\right)+\zeta_{5} \Lambda^{2}\right\} \chi_{1}^{+q} \chi_{1}^{-q}+\left\{\left(\frac{\lambda_{2} w\left(u^{2}-u^{\prime 2}\right)}{u}+\frac{\zeta_{6} u^{\prime 2} w^{\prime 2} w}{2 u\left(u^{\prime 2}-w^{2}\right)}\right) \phi_{13}^{+q} \phi_{31}^{-q}\right. \\
& +\frac{\left(u^{\prime 2}-u^{2}\right)\left(2 \lambda_{2}\left(u^{2}-w^{2}\right)\left(u^{\prime 2}-w^{2}\right)+\zeta_{6} w^{2} w^{\prime 2}\right)}{2 \sqrt{2} u\left(u^{\prime 2}-w^{2}\right) \Lambda} \phi_{13}^{+q} \sigma_{13}^{-q}+\frac{\zeta_{6} u w^{\prime}}{2} \phi_{13}^{+q} \chi_{1}^{-q}+\frac{\zeta_{5} w^{\prime} \Lambda}{2 \sqrt{2}} \sigma_{13}^{+q} \chi_{1}^{-q} \\
& \left.+\frac{\left(u^{\prime 2}-u^{2}\right) w\left(2 \lambda_{2}\left(u^{2}-w^{2}\right)\left(u^{\prime 2}-w^{2}\right)+\zeta_{6} w^{2} w^{\prime 2}\right)}{2 \sqrt{2} u^{2}\left(u^{\prime 2}-w^{2}\right) \Lambda} \phi_{31}^{+q} \sigma_{13}^{-q}+\frac{\zeta_{6} w w^{\prime}}{2} \phi_{31}^{+q} \chi_{1}^{-q}+\text { H.c. }\right\} .
\end{aligned}
$$

$$
\begin{aligned}
& V_{\text {mass }}^{(q+1) \text {-charged }}=\frac{\zeta_{6} u^{\prime 2} w^{\prime 2}}{2\left(u^{\prime 2}-w^{2}\right)} \phi_{23}^{q+1} \phi_{23}^{-(q+1)}+\frac{\zeta_{6} w^{2} w^{\prime 2}}{2\left(u^{\prime 2}-w^{2}\right)} \phi_{32}^{q+1} \phi_{32}^{-(q+1)}+\frac{1}{2} \zeta_{6}\left(u^{\prime 2}-w^{2}\right) \chi_{2}^{(q+1)} \chi_{2}^{-(q+1)} \\
& +\left\{\frac{\zeta_{6} u^{\prime} w w^{\prime 2}}{2\left(u^{\prime 2}-w^{2}\right)} \phi_{23}^{q+1} \phi_{32}^{-(q+1)}+\frac{\zeta_{6} u^{\prime} w^{\prime}}{2} \phi_{23}^{q+1} \chi_{2}^{-(q+1)}+\frac{\zeta_{6} w w^{\prime}}{2} \phi_{32}^{q+1} \chi_{2}^{-(q+1)}+\text { H.c. }\right\} .
\end{aligned}
$$

\section{B Fermion gauge-boson interactions}

The gauge interactions of fermions arise from,

$$
\mathcal{L} \supset \bar{\Psi} i \gamma^{\mu} \partial_{\mu} \Psi-g_{L} \bar{\Psi}_{L} \gamma^{\mu}\left(P_{L \mu}^{C C}+P_{L \mu}^{N C}\right) \Psi_{L}-g_{R} \bar{\Psi}_{R} \gamma^{\mu}\left(P_{R \mu}^{C C}+P_{R \mu}^{N C}\right) \Psi_{R},
$$

where $\Psi_{L}$ and $\Psi_{R}$ run on all left-handed and right-handed fermion multiplets, respectively, and $P_{L, R}^{C C}=\sum_{n=1,2,4,5,6,7} T_{n L, R} A_{n L, R}, P_{L, R}^{N C}=T_{3 L, R} A_{3 L, R}+T_{8 L, R} A_{8 L, R}+\frac{g_{X}}{g_{L, R}} X_{\Psi_{L, R}} B$.

The interactions of the physical charged gauge bosons with fermions are

$\mathcal{L}_{C C}=J_{1 W}^{-\mu} W_{1 \mu}^{+}+J_{2 W}^{-\mu} W_{2 \mu}^{+}+J_{1 X}^{-q \mu} X_{1 \mu}^{q}+J_{2 X}^{-q \mu} X_{2 \mu}^{q}+J_{1 Y}^{-(q+1) \mu} Y_{1 \mu}^{q+1}+J_{2 Y}^{-(q+1) \mu} Y_{2 \mu}^{q+1}+$ H.c., where the charged currents take the form,

$$
\begin{aligned}
J_{1 W}^{-\mu} & =-\frac{g_{L} c_{\xi}}{\sqrt{2}}\left(\bar{\nu}_{a L} \gamma^{\mu} e_{a L}+\bar{u}_{a L} \gamma^{\mu} d_{a L}\right)+\frac{g_{R} s_{\xi}}{\sqrt{2}}\left(\bar{\nu}_{a R} \gamma^{\mu} e_{a R}+\bar{u}_{a R} \gamma^{\mu} d_{a R}\right), \\
J_{2 W}^{-\mu} & =-\frac{g_{L} \xi_{\xi}}{\sqrt{2}}\left(\bar{\nu}_{a L} \gamma^{\mu} e_{a L}+\bar{u}_{a L} \gamma^{\mu} d_{a L}\right)-\frac{g_{R} c_{\xi}}{\sqrt{2}}\left(\bar{\nu}_{a R} \gamma^{\mu} e_{a R}+\bar{u}_{a R} \gamma^{\mu} d_{a R}\right), \\
J_{1 X}^{-q \mu} & =-\frac{g_{L} c_{\xi_{1}}}{\sqrt{2}}\left(\bar{N}_{a L} \gamma^{\mu} \nu_{a L}-\bar{d}_{\alpha L} \gamma^{\mu} J_{\alpha L}+\bar{J}_{3 L} \gamma^{\mu} u_{3 L}\right)+\frac{g_{R} \xi_{\xi_{1}}}{\sqrt{2}}\left(\bar{N}_{a R} \gamma^{\mu} \nu_{a R}-\bar{d}_{\alpha R} \gamma^{\mu} J_{\alpha R}+\bar{J}_{3 R} \gamma^{\mu} u_{3 R}\right), \\
J_{2 X}^{-q \mu} & =-\frac{g_{L} s_{\xi_{1}}}{\sqrt{2}}\left(\bar{N}_{a L} \gamma^{\mu} \nu_{a L}-\bar{d}_{\alpha L} \gamma^{\mu} J_{\alpha L}+\bar{J}_{3 L} \gamma^{\mu} u_{3 L}\right)-\frac{g_{R} c_{\xi_{1}}}{\sqrt{2}}\left(\bar{N}_{a R} \gamma^{\mu} \nu_{a R}-\bar{d}_{\alpha R} \gamma^{\mu} J_{\alpha R}+\bar{J}_{3 R} \gamma^{\mu} u_{3 R}\right), \\
J_{1 Y}^{-(q+1) \mu} & =-\frac{g_{L} c_{\xi_{2}}}{\sqrt{2}}\left(\bar{N}_{a L} \gamma^{\mu} e_{a L}+\bar{u}_{\alpha L} \gamma^{\mu} J_{\alpha L}+\bar{J}_{3 L} \gamma^{\mu} d_{3 L}\right)+\frac{g_{R} s_{\xi_{2}}}{\sqrt{2}}\left(\bar{N}_{a R} \gamma^{\mu} e_{a R}+\bar{u}_{\alpha R} \gamma^{\mu} J_{\alpha R}+\bar{J}_{3 R} \gamma^{\mu} d_{3 R}\right), \\
J_{2 Y}^{-(q+1) \mu} & =-\frac{g_{L} \xi_{\xi_{2}}}{\sqrt{2}}\left(\bar{N}_{a L} \gamma^{\mu} e_{a L}+\bar{u}_{\alpha L} \gamma^{\mu} J_{\alpha L}+\bar{J}_{3 L} \gamma^{\mu} d_{3 L}\right)-\frac{g_{R} \xi_{2}}{\sqrt{2}}\left(\bar{N}_{a R} \gamma^{\mu} e_{a R}+\bar{u}_{\alpha R} \gamma^{\mu} J_{\alpha R}+\bar{J}_{3 R} \gamma^{\mu} d_{3 R}\right),
\end{aligned}
$$

The interactions of the physical neutral gauge bosons with fermions are obtained by

$$
\begin{aligned}
\mathcal{L}_{N C}= & -g_{L} \bar{\Psi}_{L} \gamma^{\mu} P_{L \mu}^{N C} \Psi_{L}-g_{R} \bar{\Psi}_{R} \gamma^{\mu} P_{R \mu}^{N C} \Psi_{R} \\
= & -e Q(f) \bar{f} \gamma^{\mu} f A_{\mu}-\frac{g_{L}}{2 c_{W}} \bar{f} \gamma^{\mu}\left[g_{V}^{Z_{L}}(f)-g_{A}^{Z_{L}}(f) \gamma_{5}\right] f Z_{L \mu}-\frac{g_{L}}{2 c_{W}} \bar{f} \gamma^{\mu}\left[g_{V}^{\mathcal{Z}^{\prime}{ }^{L}}(f)-g_{A}^{\mathcal{Z}^{\prime}{ }_{L}}(f) \gamma_{5}\right] f \mathcal{Z}^{\prime}{ }_{L \mu} \\
& -\frac{g_{L}}{2 c_{W}} \bar{f} \gamma^{\mu}\left[g_{V}^{\mathcal{Z}_{R}}(f)-g_{A}^{\mathcal{Z}_{R}}(f) \gamma_{5}\right] f \mathcal{Z}_{R \mu}-\frac{g_{L}}{2 c_{W}} \bar{f} \gamma^{\mu}\left[g_{V}^{\mathcal{Z}_{R}^{\prime}}(f)-g_{A}^{\mathcal{Z}_{R}^{\prime}}(f) \gamma_{5}\right] f \mathcal{Z}_{R \mu}^{\prime},
\end{aligned}
$$




\begin{tabular}{|c|c|}
\hline$f$ & $g_{V}^{Z_{L}}(f)$ \\
\hline$g_{V}^{Z_{L}}\left(\nu_{a}\right)$ & $\frac{\left\{t_{R}^{2}\left[3+\left(1+t_{R}\right) t_{X}(3+\sqrt{3} \beta)\right]+t_{X}^{2}\left[3+3 \beta^{2}+t_{R}^{2}\left(3 \beta^{2}-3-2 \sqrt{3} \beta\right)\right]\right\} c_{W}^{2}}{6\left[t_{R}^{2}+t_{X}^{2}+\left(1+t_{R}^{2}\right) t_{X}^{2} \beta^{2}\right]}$ \\
\hline$g_{A}^{Z_{L}}\left(\nu_{a}\right)$ & $\frac{\left\{t_{R}^{2}\left[3-\left(t_{R}-1\right) t_{X}(3+\sqrt{3} \beta)\right]+3\left(1+t_{R}^{2}\right) t_{X}^{2}\left(1+\beta^{2}\right)\right\} c_{W}^{2}}{6\left[t_{R}^{2}+t_{X}^{2}+\left(1+t_{R}^{2}\right) t_{X}^{2} \beta^{2}\right]}$ \\
\hline$g_{V}^{Z_{L}}\left(e_{a}\right)$ & $-\frac{\left\{t_{R}^{2}\left[3-\left(1+t_{R}\right) t_{X}(3+\sqrt{3} \beta)\right]+t_{X}^{2}\left[3+3 \beta^{2}+t_{R}^{2}\left(-3+2 \sqrt{3} \beta+3 \beta^{2}\right)\right]\right\} c_{W}^{2}}{6\left[t_{R}^{2}+t_{X}^{2}+\left(1+t_{R}^{2}\right) t_{X}^{2} \beta^{2}\right]}$ \\
\hline$g_{A}^{Z_{L}}\left(e_{a}\right)$ & $-\frac{\left\{t_{R}^{2}\left[3+\left(t_{R}-1\right) t_{X}(3+\sqrt{3} \beta]+3\left(t+t_{R}^{2}\right) t_{X}^{2}\left(1+\beta^{2}\right)\right\} c_{W}^{2}\right.}{6\left[t_{R}^{2}+t_{X}^{2}+\left(1+t_{R}^{2}\right) t_{X}^{2} \beta^{2}\right]}$ \\
\hline$g_{V}^{Z_{L}}\left(N_{a}\right)$ & $\frac{t_{X} t_{R}^{2}\left\{4 \sqrt{3} \beta t_{X}+\left(1+t_{R}\right)(3+\sqrt{3} \beta)\right\} c_{W}^{2}}{6\left[t_{R}^{2}+t_{X}^{2}+\left(1+t_{R}^{2}\right) t_{X}^{2} \beta^{2}\right]}$ \\
\hline$g_{A}^{Z_{L}}\left(N_{a}\right)$ & $-\frac{\left(t_{R}-1\right) t_{R}^{2} t_{X}(3+\sqrt{3} \beta) c_{w}^{2}}{6\left[t_{R}^{2}+t_{X}^{2}+\left(1+t_{R}^{2}\right) t_{X}^{2} \beta^{2}\right]}$ \\
\hline$g_{V}^{Z_{L}}\left(u_{\alpha}\right)$ & $-\frac{\left\{3 t_{R}^{2}+\left(1+t_{R}\right) t_{X} t_{R}^{2}(1+\sqrt{3} \beta)+t_{X}^{2}\left[3+3 \beta^{2}+t_{R}^{2}\left(3 \beta^{2}+2 \sqrt{3} \beta-3\right)\right]\right\} c_{W}^{2}}{6\left[t_{R}^{2}+t_{X}^{2}+\left(1+t_{R}^{2}\right) t_{X}^{2} \beta^{2}\right]}$ \\
\hline$g_{A}^{Z_{L}}\left(u_{\alpha}\right)$ & $-\frac{\left\{3 t_{R}^{2}-\left(t_{R}-1\right) t_{R}^{2} t_{X}(1+\sqrt{3} \beta)+3\left(1+t_{R}^{2}\right) t_{X}^{2}\left(1+\beta^{2}\right)\right\} c_{w}^{2}}{6\left[t_{R}^{2}+t_{X}^{2}+\left(1+t_{R}^{2}\right) t_{X}^{2} \beta^{2}\right]}$ \\
\hline$g_{V}^{Z_{L}}\left(u_{3}\right)$ & $\frac{\left\{3 t_{R}^{2}+t_{R}^{2}\left(1+t_{R}\right) t_{X}(\sqrt{3} \beta-1)+t_{X}^{2}\left[3+3 \beta^{2}+t_{R}^{2}\left(3 \beta^{2}-3-2 \sqrt{3} \beta\right)\right]\right\} c_{W}}{6\left[t_{R}^{2}+t_{X}^{2}+\left(1+t_{R}^{2}\right) t_{X}^{2} \beta^{2}\right]}$ \\
\hline$g_{A}^{Z_{L}}\left(u_{3}\right)$ & $\frac{\left\{3 t_{R}^{2}-\left(t_{R}-1\right) t_{R}^{2} t_{X}(\sqrt{3} \beta-1)+3\left(1+t_{R}^{2}\right) t_{X}^{2}\left(1+\beta^{2}\right)\right\} c_{W}^{2}}{6\left[t_{R}^{2}+t_{X}^{2}+\left(1+t_{R}^{2}\right) t_{X}^{2} \beta^{2}\right]}$ \\
\hline$g_{V}^{Z_{L}}\left(d_{\alpha}\right)$ & $\frac{\left\{3 t_{R}^{2}-t_{R}^{2}\left(1+t_{R}\right) t_{X}(1+\sqrt{3} \beta)+t_{X}^{2}\left[3+3 \beta^{2}+t_{R}^{2}\left(3 \beta^{2}-2 \sqrt{3} \beta-3\right)\right]\right\} c_{W}^{2}}{6\left[t_{R}^{2}+t_{X}^{2}+\left(1+t_{R}^{2}\right) t_{X}^{2} \beta^{2}\right]}$ \\
\hline$g_{A}^{Z_{L}}\left(d_{\alpha}\right)$ & $\frac{\left\{3 t_{R}^{2}+\left(t_{R}-1\right) t_{R}^{2} t_{X}(1+\sqrt{3} \beta)+3\left(1+t_{R}^{2}\right) t_{X}^{2}\left(1+\beta^{2}\right)\right\} c_{W}^{2}}{6\left[t_{R}^{2}+t_{X}^{2}+\left(1+t_{R}^{2}\right) t_{X}^{2} \beta^{2}\right]}$ \\
\hline$g_{V}^{Z_{L}}\left(d_{3}\right)$ & $\frac{\left\{-3 t_{R}^{2}+t_{R}^{2}\left(1+t_{R}\right) t_{X}(\sqrt{3} \beta-1)-t_{X}^{2}\left[3+3 \beta^{2}+t_{R}^{2}\left(3 \beta^{2}+2 \sqrt{3} \beta-3\right)\right]\right\} c_{W}^{2}}{6\left[t_{R}^{2}+t_{X}^{2}+\left(1+t_{R}^{2}\right) \beta^{2} t_{X}^{2}\right]}$ \\
\hline$g_{A}^{Z_{L}}\left(d_{3}\right)$ & $\frac{\left\{-3 t_{R}^{2}+\left(1-t_{R}\right) t_{R}^{2} t_{X}(\sqrt{3} \beta-1)-3 t_{X}^{2}\left(1+t_{R}^{2}\right)\left(1+\beta^{2}\right)\right\} c_{W}^{2}}{6\left[t_{R}^{2}+t_{X}^{2}+\left(1+t_{R}^{2}\right) \beta^{2} t_{X}^{2}\right]}$ \\
\hline$g_{V}^{Z_{L}}\left(J_{\alpha}\right)$ & $-\frac{t_{X} t_{R}^{2}\left[1+t_{R}+\sqrt{3} t_{R} \beta+\sqrt{3}\left(1-4 t_{X}\right) \beta\right] c_{W}^{2}}{6\left[t_{R}^{2}+t_{X}^{2}+\left(1+t_{R}^{2}\right) t_{X}^{2} \beta^{2}\right]}$ \\
\hline$g_{A}^{Z_{L}}\left(J_{\alpha}\right)$ & $\frac{\left(t_{R}-1\right) t_{R}^{2} t_{X}(1+\sqrt{3} \beta) c_{W}^{2}}{6\left[t_{R}^{2}+t_{X}^{2}+\left(1+t_{R}^{2}\right) t_{X}^{2} \beta^{2}\right]}$ \\
\hline$g_{V}^{Z_{L}}\left(J_{3}\right)$ & $\frac{t_{R}^{2} t_{X}\left[4 \sqrt{3} t_{X} \beta+\left(1+t_{R}\right)(\sqrt{3} \beta-1)\right] c_{W}^{2}}{6\left[t_{R}^{2}+t_{X}^{2}+\left(1+t_{R}^{2}\right) t_{X}^{2} \beta^{2}\right]}$ \\
\hline$g_{A}^{Z_{L}}\left(J_{3}\right)$ & $\frac{\left(t_{R}-1\right) t_{R}^{2} t_{X}(\sqrt{3} \beta-1) c_{W}^{2}}{6\left[t_{R}^{2}+t_{X}^{2}+\left(1+t_{R}^{2}\right) t_{X}^{2} \beta^{2}\right]}$ \\
\hline
\end{tabular}

Table 2. The couplings of $Z_{L}$ with fermions.

where $f$ stands for every all the fermion fields, and $e=g_{L} s_{W}$. The vector and axial-vector couplings $g_{V, A}^{Z_{L}, \mathcal{Z}_{L}^{\prime}, \mathcal{Z}_{R}, \mathcal{Z}_{R}^{\prime}}(f)$ are collected in tables 2, 3, 4, and 5. Note that at high energy $g_{L}=g_{R}$, i.e. $t_{X}=t_{R}$, due to the left-right symmetry. However, at the low energy, such relation does not hold anymore. Therefore, the couplings we provide are general, depending on both $t_{X}$ and $t_{R}$. 


\begin{tabular}{|c|c|}
\hline$g_{V}^{Z_{L}^{\prime}}\left(\nu_{a}\right)$ & $\frac{\left\{-\sqrt{3}\left(t_{R}^{2}+t_{X}^{2}\right)-3 \beta t_{X} t_{R}^{2}\left(1+t_{R}-t_{X}\right)-\sqrt{3} \beta^{2} t_{X}\left(1+t_{R}\right)\left(t_{R}^{2}+t_{X}-t_{R} t_{X}\right)\right\}}{\zeta_{1}^{-1} t_{R}^{-1} t_{X}^{-1} c_{W}^{-1} t_{W} 6\left[t_{R}^{2}+t_{X}^{2}+\left(1+t_{R}^{2}\right) t_{X}^{2} \beta^{2}\right]}$ \\
\hline$g_{A}^{Z_{L}^{\prime}}\left(\nu_{a}\right)$ & $\frac{\left\{t_{R}^{3} t_{X} \beta(3+\sqrt{3} \beta)-\sqrt{3} t_{X}^{2}\left(1+\beta^{2}\right)-t_{R}^{2}\left[\sqrt{3}+t_{X}\left(1+t_{X}\right) \beta(3+\sqrt{3} \beta)\right]\right\}}{\zeta_{1}^{-1} t_{R}^{-1} t_{X}^{-1} c_{W}^{-1} t_{W} 6\left[t_{R}^{2}+t_{X}^{2}+\left(1+t_{R}^{2}\right) t_{X}^{2} \beta^{2}\right]}$ \\
\hline$g_{V}^{Z_{L}^{\prime}}\left(e_{a}\right)$ & $\frac{\left\{-\sqrt{3} t_{R}^{2}-t_{R}^{2}\left(1+t_{R}\right) t_{X} \beta(3+\sqrt{3} \beta)+t_{X}^{2}\left[t_{R}^{2} \beta(-3+\sqrt{3} \beta)-\sqrt{3}\left(1+\beta^{2}\right)\right]\right\}}{\zeta_{1}^{-1} t_{R}^{-1} t_{X}^{-1} c_{W}^{-1} t_{W} 6\left[t_{R}^{2}+t_{X}^{2}+\left(1+t_{R}^{2}\right) t_{X}^{2} \beta^{2}\right]}$ \\
\hline$g_{A}^{Z_{L}^{\prime}}\left(e_{a}\right)$ & $\frac{\left\{-\sqrt{3}\left(t_{R}^{2}+t_{X}^{2}\right)+3 \beta t_{X} t_{R}^{2}\left(-1+t_{R}+t_{X}\right)-\sqrt{3} \beta^{2} t_{X}\left[t_{X}+t_{R}^{2}\left(1-t_{R}+t_{X}\right)\right]\right\}}{\zeta_{1}^{-1} t_{R}^{-1} t_{X}^{-1} c_{W}^{-1} t_{W} 6\left[t_{R}^{2}+t_{X}^{2}+\left(1+t_{R}^{2}\right) t_{X}^{2} \beta^{2}\right]}$ \\
\hline$g_{V}^{Z_{L}^{\prime}}\left(N_{a}\right)$ & $\frac{\left\{2 \sqrt{3} t_{R}^{2}-\beta t_{X} t_{R}^{2}\left(1+t_{R}\right)(3+\sqrt{3} \beta)+2 \sqrt{3} t_{X}^{2}\left[1-\left(t_{R}^{2}-1\right) \beta^{2}\right]\right\}}{\zeta_{1}^{-1} t_{R}^{-1} t_{X}^{-1} c_{W}^{-1} t_{W} 6\left[t_{R}^{2}+t_{X}^{2}+\left(1+t_{R}^{2}\right) t_{X}^{2} \beta^{2}\right]}$ \\
\hline$g_{A}^{Z_{L}^{\prime}}\left(N_{a}\right)$ & $\frac{\left\{2 \sqrt{3} t_{R}^{2}+\beta t_{X} t_{R}^{2}\left(t_{R}-1\right)(3+\sqrt{3} \beta)+2 \sqrt{3} t_{X}^{2}\left[1+\left(1+t_{R}^{2}\right) \beta^{2}\right]\right\}}{\zeta_{1}^{-1} t_{R}^{-1} t_{X}^{-1} c_{W}^{-1} t_{W} 6\left[t_{R}^{2}+t_{X}^{2}+\left(1+t_{R}^{2}\right) t_{X}^{2} \beta^{2}\right]}$ \\
\hline$g_{V}^{Z_{L}^{\prime}}\left(u_{\alpha}\right)$ & $\frac{\left\{-\sqrt{3} t_{R}^{2}+\beta t_{X} t_{R}^{2}\left(t_{R}+1\right)(\sqrt{3} \beta+1)+t_{X}^{2}\left[t_{R}^{2} \beta(-3+\sqrt{3} \beta)-\sqrt{3}\left(1+\beta^{2}\right)\right]\right\}}{\zeta_{1}^{-1} t_{R}^{-1} t_{X}^{-1} c_{W}^{-1} t_{W} 6\left[t_{R}^{2}+t_{X}^{2}+\left(1+t_{R}^{2}\right) t_{X}^{2} \beta^{2}\right]}$ \\
\hline$g_{A}^{Z_{L}^{\prime}}\left(u_{\alpha}\right)$ & $\frac{\left\{-\sqrt{3} t_{R}^{2}-\beta t_{X} t_{R}^{2}\left(t_{R}-1\right)(1+\sqrt{3} \beta)-t_{X}^{2}\left[\beta t_{R}^{2}(\sqrt{3} \beta-3)+\sqrt{3}\left(1+\beta^{2}\right)\right]\right\}}{\zeta_{1}^{-1} t_{R}^{-1} t_{X}^{-1} c_{W}^{-1} t_{W} 6\left[t_{R}^{2}+t_{X}^{2}+\left(1+t_{R}^{2}\right) t_{X}^{2} \beta^{2}\right]}$ \\
\hline$g_{V}^{Z_{L}^{\prime}}\left(u_{3}\right)$ & $\frac{\left\{-\sqrt{3} t_{R}^{2}-\beta t_{X} t_{R}^{2}\left(t_{R}+1\right)(\sqrt{3} \beta-1)+t_{X}^{2}\left[t_{R}^{2} \beta(3+\sqrt{3} \beta)-\sqrt{3}\left(1+\beta^{2}\right)\right]\right\}}{\zeta_{1}^{-1} t_{R}^{-1} t_{X}^{-1} c_{W}^{-1} t_{W} 6\left[t_{R}^{2}+t_{X}^{2}+\left(1+t_{R}^{2}\right) t_{X}^{2} \beta^{2}\right]}$ \\
\hline$g_{A}^{Z_{L}^{\prime}}\left(u_{3}\right)$ & $\frac{\left\{-\sqrt{3}\left(t_{R}^{2}+t_{X}^{2}\right)-\beta t_{X} t_{R}^{2}\left(t_{R}-1+3 t_{X}\right)-\sqrt{3} t_{X}\left[t_{X}+t_{R}^{2}\left(1+t_{X}-t_{R}\right)\right] \beta^{2}\right\}}{\zeta_{1}^{-1} t_{R}^{-1} t_{X}^{-1} c_{W}^{-1} t_{W} 6\left[t_{R}^{2}+t_{X}^{2}+\left(1+t_{R}^{2}\right) t_{X}^{2} \beta^{2}\right]}$ \\
\hline$g_{V}^{Z_{L}^{\prime}}\left(d_{\alpha}\right)$ & $\frac{\left\{-\sqrt{3} t_{R}^{2}+\beta t_{X} t_{R}^{2}\left(t_{R}+1\right)(\sqrt{3} \beta+1)+t_{X}^{2}\left[t_{R}^{2} \beta(3+\sqrt{3} \beta)-\sqrt{3}\left(1+\beta^{2}\right)\right]\right\}}{\zeta_{1}^{-1} t_{R}^{-1} t_{X}^{-1} c_{W}^{-1} t_{W} 6\left[t_{R}^{2}+t_{X}^{2}+\left(1+t_{R}^{2}\right) t_{X}^{2} \beta^{2}\right]}$ \\
\hline$g_{A}^{Z_{L}^{\prime}}\left(d_{\alpha}\right)$ & $\frac{\left\{-\sqrt{3} t_{R}^{2}-\beta t_{X} t_{R}^{2}\left(t_{R}-1\right)(1+\sqrt{3} \beta)-t_{X}^{2}\left[\beta t_{R}^{2}(3+\sqrt{3} \beta)+\sqrt{3}\left(1+\beta^{2}\right)\right]\right\}}{\zeta_{1}^{-1} t_{R}^{-1} t_{X}^{-1} c_{W}^{-1} t_{W} 6\left[t_{R}^{2}+t_{X}^{2}+\left(1+t_{R}^{2}\right) t_{X}^{2} \beta^{2}\right]}$ \\
\hline$g_{V}^{Z_{L}^{\prime}}\left(d_{3}\right)$ & $\frac{\left\{-\sqrt{3} t_{R}^{2}-\beta t_{X} t_{R}^{2}\left(t_{R}+1\right)(\sqrt{3} \beta-1)+t_{X}^{2}\left[t_{R}^{2} \beta(-3+\sqrt{3} \beta)-\sqrt{3}\left(1+\beta^{2}\right)\right]\right\}}{\zeta_{1}^{-1} t_{R}^{-1} t_{X}^{-1} c_{W}^{-1} t_{W} 6\left[t_{R}^{2}+t_{X}^{2}+\left(1+t_{R}^{2}\right) t_{X}^{2} \beta^{2}\right]}$ \\
\hline$g_{A}^{Z_{L}^{\prime}}\left(d_{3}\right)$ & $\frac{\left\{-\sqrt{3}\left(t_{R}^{2}+t_{X}^{2}\right)+\beta t_{X} t_{R}^{2}\left(1-t_{R}+3 t_{X}\right)-\sqrt{3} t_{X}\left[t_{X}+t_{R}^{2}\left(1+t_{X}-t_{R}\right)\right] \beta^{2}\right\}}{\zeta_{1}^{-1} t_{R}^{-1} t_{X}^{-1} c_{W}^{-1} t_{W} 6\left[t_{R}^{2}+t_{X}^{2}+\left(1+t_{R}^{2}\right) t_{X}^{2} \beta^{2}\right]}$ \\
\hline$g_{V}^{Z_{L}^{\prime}}\left(J_{\alpha}\right)$ & $\frac{\left\{2 \sqrt{3} t_{R}^{2}+\beta t_{X} t_{R}^{2}\left(t_{R}+1\right)(\sqrt{3} \beta+1)+2 \sqrt{3} t_{X}^{2}\left[1-\left(t_{R}^{2}-1\right) \beta^{2}\right]\right\}}{\zeta_{1}^{-1} t_{R}^{-1} t_{X}^{-1} c_{W}^{-1} t_{W} 6\left[t_{R}^{2}+t_{X}^{2}+\left(1+t_{R}^{2}\right) t_{X}^{2} \beta^{2}\right]}$ \\
\hline$g_{A}^{Z_{L}^{\prime}}\left(J_{\alpha}\right)$ & $\frac{\left\{2 \sqrt{3} t_{R}^{2}-\beta t_{X} t_{R}^{2}\left(t_{R}-1\right)(\sqrt{3} \beta+1)+2 \sqrt{3} t_{X}^{2}\left[1+\beta^{2}\left(1+t_{R}^{2}\right)\right]\right\}}{\zeta_{1}^{-1} t_{R}^{-1} t_{X}^{-1} c_{W}^{-1} t_{W} 6\left[t_{R}^{2}+t_{X}^{2}+\left(1+t_{R}^{2}\right) t_{X}^{2} \beta^{2}\right]}$ \\
\hline$g_{V}^{Z_{L}^{\prime}}\left(J_{3}\right)$ & $\frac{\left\{2 \sqrt{3} t_{R}^{2}-\beta t_{X} t_{R}^{2}\left(t_{R}+1\right)(\sqrt{3} \beta-1)+2 \sqrt{3} t_{X}^{2}\left[1-\left(t_{R}^{2}-1\right) \beta^{2}\right]\right\}}{\zeta_{1}^{-1} t_{R}^{-1} t_{X}^{-1} c_{W}^{-1} t_{W} 6\left[t_{R}^{2}+t_{X}^{2}+\left(1+t_{R}^{2}\right) t_{X}^{2} \beta^{2}\right]}$ \\
\hline$g_{A}^{Z_{L}^{\prime}}\left(J_{3}\right)$ & $\frac{\left\{2 \sqrt{3} t_{R}^{2}+\beta t_{X} t_{R}^{2}\left(t_{R}-1\right)(\sqrt{3} \beta-1)+2 \sqrt{3} t_{X}^{2}\left[1+\beta^{2}\left(1+t_{R}^{2}\right)\right]\right\}}{\zeta_{1}^{-1} t_{R}^{-1} t_{X}^{-1} c_{W}^{-1} t_{W} 6\left[t_{R}^{2}+t_{X}^{2}+\left(1+t_{R}^{2}\right) t_{X}^{2} \beta^{2}\right]}$ \\
\hline
\end{tabular}

Table 3. The couplings of $Z_{L}^{\prime}$ with fermions. 


\begin{tabular}{|c|c|}
\hline$f$ & $g_{V}^{\mathcal{Z}_{\mathcal{R}}}(f)$ \\
\hline$\nu_{a}$ & $-\frac{\left\{c_{\epsilon_{2}} t_{R} \zeta_{1}^{-1}\left[3 \zeta^{-2}-\sqrt{3} \beta t_{X}^{2}+t_{X}\left(1+t_{R}\right)(3+\sqrt{3} \beta)\right]+s_{\epsilon_{2}} \zeta_{1}^{-2}\left[\sqrt{3} t_{R}^{2}+t_{X} \beta(3+\sqrt{3} \beta)\left(1+t_{R}\right)\right]\right\} c_{W}}{6 \zeta^{-1} \zeta_{1}^{-2}}$ \\
\hline$e_{a}$ & $-\frac{\left\{c_{\epsilon_{2}} t_{R} \zeta_{1}^{-1}\left[-3 \zeta^{-2}-\sqrt{3} \beta t_{X}^{2}+t_{X}\left(1+t_{R}\right)(3+\sqrt{3} \beta)\right]+s_{\epsilon_{2}} \zeta_{1}^{-2}\left[\sqrt{3} t_{R}^{2}+t_{X} \beta(3+\sqrt{3} \beta)\left(1+t_{R}\right)\right] c_{W}\right.}{6 \zeta^{-1} \zeta_{1}^{-2}}$ \\
\hline$N_{a}$ & $-\frac{\left\{c_{\epsilon_{2}} t_{R} t_{X} \zeta_{1}^{-1}\left[2 \sqrt{3} t_{X} \beta+\left(t_{R}+1\right)(3+\sqrt{3} \beta)\right]+s_{\epsilon_{2}} \zeta_{1}^{-2}\left[-2 \sqrt{3} t_{R}^{2}+t_{X} \beta(3+\sqrt{3} \beta)\left(t_{R}+1\right)\right]\right\} c_{W}}{6 \zeta^{-1} \zeta_{1}^{-2}}$ \\
\hline$u_{\alpha}$ & $\frac{\left\{c_{\epsilon_{2}} t_{R} \zeta_{1}^{-1}\left[3 \zeta^{-2}+t_{R}\left(1+t_{X}\right)+\sqrt{3} \beta t_{X}\left(1+t_{R}+t_{X}\right)\right]+s_{\epsilon_{2}} \zeta_{1}^{-2}\left[-\sqrt{3} t_{R}^{2}+t_{X} \beta(1+\sqrt{3} \beta)\left(1+t_{R}\right)\right]\right\} c_{W}}{6 \zeta^{-1} \zeta_{1}^{-2}}$ \\
\hline$u_{3}$ & $\frac{\left\{c_{\epsilon_{2}} t_{R} \zeta_{1}^{-1}\left[-3 \zeta^{-2}+t_{X}\left(t_{R}+1\right)+\sqrt{3} t_{X}\left(t_{X}-t_{R}-1\right) \beta\right]-s_{\epsilon_{2}} \zeta_{1}^{-2}\left[\sqrt{3} t_{R}^{2}+t_{X} \beta(\sqrt{3} \beta-1)\left(t_{R}+1\right)\right]\right\} c_{W}}{6 \zeta^{-1} \zeta_{1}^{-2}}$ \\
\hline$d_{\alpha}$ & $\frac{\left\{c_{\epsilon_{2}} t_{R} \zeta_{1}^{-1}\left[-3 \zeta^{-2}+t_{X}\left(t_{R}+1\right)+\sqrt{3} \beta t_{X}\left(t_{X}+t_{R}+1\right)\right]+s_{\epsilon_{2}} \zeta_{1}^{-2}\left[-\sqrt{3} t_{R}^{2}+t_{X} \beta(\sqrt{3} \beta+1)\left(t_{R}+1\right)\right]\right\} c_{W}}{6 \zeta^{-1} \zeta_{1}^{-2}}$ \\
\hline$d_{3}$ & $\frac{\left\{c_{\epsilon_{2}} t_{R} \zeta_{1}^{-1}\left[3 \zeta^{-2}+t_{X}\left(t_{R}+1\right)+\sqrt{3} \beta t_{X}\left(t_{X}-t_{R}-1\right)\right]-s_{\epsilon_{2}} \zeta_{1}^{-2}\left[\sqrt{3} t_{R}^{2}+t_{X} \beta(\sqrt{3} \beta-1)\left(t_{R}+1\right)\right]\right\} c_{W}}{6 \zeta^{-1} \zeta_{1}^{-2}}$ \\
\hline$J_{\alpha}$ & $\frac{\left\{c_{\epsilon_{2}} t_{R} t_{X} \zeta_{1}^{-1}\left[1+t_{R}+\sqrt{3} \beta\left(1+t_{R}-2 t_{X}\right)\right]+s_{\epsilon_{2}} \zeta_{1}^{-2}\left[2 \sqrt{3} t_{R}^{2}+t_{X} \beta(\sqrt{3} \beta+1)\left(t_{R}+1\right)\right] c_{W}\right.}{6 \zeta^{-1} \zeta_{1}^{-2}}$ \\
\hline$J_{3}$ & $\frac{\left\{c_{\epsilon_{2}} t_{R} t_{X} \zeta_{1}^{-1}\left[1+t_{R}-\sqrt{3} t_{R} \beta-\sqrt{3} \beta\left(1+2 t_{X}\right)\right]+s_{\epsilon_{2}} \zeta_{1}^{-2}\left[2 \sqrt{3} t_{R}^{2}+t_{X} \beta(1-\sqrt{3} \beta)\left(t_{R}+1\right)\right] c_{W}\right.}{6 \zeta^{-1} \zeta_{1}^{-2}}$ \\
\hline$f$ & $g_{A}^{\mathcal{Z}_{\mathcal{R}}}(f)$ \\
\hline$\nu_{a}$ & $\frac{\left\{c_{\epsilon_{2}} t_{R} \zeta_{1}^{-1}\left[3 \zeta^{-2}-\sqrt{3} \beta t_{X}^{2}+t_{X}\left(t_{R}-1\right)(3+\sqrt{3} \beta)\right]+s_{\epsilon_{2}} \zeta_{1}^{-2}\left[\sqrt{3} t_{R}^{2}+t_{X} \beta(3+\sqrt{3} \beta)\left(t_{R}-1\right)\right]\right\} c_{W}}{6 \zeta^{-1} \zeta_{1}^{-2}}$ \\
\hline$e_{a}$ & $\frac{\left\{-c_{\epsilon_{2}} t_{R} \zeta_{1}^{-1}\left[3 \zeta^{-2}+\sqrt{3} \beta t_{X}^{2}+t_{X}\left(t_{R}-1\right)(3+\sqrt{3} \beta)\right]+s_{\epsilon_{2}} \zeta_{1}^{-2}\left[\sqrt{3} t_{R}^{2}+t_{X} \beta(3+\sqrt{3} \beta)\left(t_{R}-1\right)\right]\right\} c_{W}}{6 \zeta^{-1} \zeta_{1}^{-2}}$ \\
\hline$N_{a}$ & $\frac{\left\{c_{\epsilon_{2}} t_{R} t_{X} \zeta_{1}^{-1}\left[2 \sqrt{3} t_{X} \beta+\left(t_{R}-1\right)(3+\sqrt{3} \beta)\right]+s_{\epsilon_{2}} \zeta_{1}^{-2}\left[-2 \sqrt{3} t_{R}^{2}+t_{X} \beta(3+\sqrt{3} \beta)\left(t_{R}-1\right)\right]\right\} c_{W}}{6 \zeta^{-1} \zeta_{1}^{-2}}$ \\
\hline$u_{\alpha}$ & $\frac{\left\{-c_{\epsilon_{2}} t_{R} \zeta_{1}^{-1}\left[3 \zeta^{-2}+t_{X}\left(t_{R}-1\right)+\sqrt{3} \beta t_{X}\left(-1+t_{R}+t_{X}\right)\right]+s_{\epsilon_{2}} \zeta_{1}^{-2}\left[\sqrt{3} t_{R}^{2}+t_{X} \beta(1+\sqrt{3} \beta)\left(1-t_{R}\right)\right]\right\} c_{W}}{6 \zeta^{-1} \zeta_{1}^{-2}}$ \\
\hline$u_{3}$ & $\frac{\left\{c_{\epsilon_{2}} t_{R} \zeta_{1}^{-1}\left[3 \zeta^{-2}+t_{X}\left(t_{R}-1\right)(\sqrt{3} \beta-1)-\sqrt{3} \beta t_{X}^{2}\right]+s_{\epsilon_{2}} \zeta_{1}^{-2}\left[\sqrt{3} t_{R}^{2}+t_{X} \beta(\sqrt{3} \beta-1)\left(t_{R}-1\right)\right] c_{W}\right.}{6 \zeta^{-1} \zeta_{1}^{-2}}$ \\
\hline$d_{\alpha}$ & $\frac{\left\{c_{\epsilon_{2}} t_{R} \zeta_{1}^{-1}\left[3 \zeta^{-2}+t_{X}\left(1-t_{R}\right)-\sqrt{3} \beta t_{X}\left(t_{X}+t_{R}-1\right)\right]+s_{\epsilon_{2}} \zeta_{1}^{-2}\left[\sqrt{3} t_{R}^{2}+t_{X} \beta(\sqrt{3} \beta+1)\left(1-t_{R}\right)\right]\right\} c_{W}}{6 \zeta^{-1} \zeta_{1}^{-2}}$ \\
\hline$d_{3}$ & $\frac{\left\{-c_{\epsilon_{2}} t_{R} \zeta_{1}^{-1}\left[3 \zeta^{-2}+t_{X}\left(t_{R}-1\right)+\sqrt{3} \beta t_{X}\left(t_{X}-t_{R}+1\right)\right]+s_{\epsilon_{2}} \zeta_{1}^{-2}\left[\sqrt{3} t_{R}^{2}+t_{X} \beta(1-\sqrt{3} \beta)\left(1-t_{R}\right)\right]\right\} c_{W}}{6 \zeta^{-1} \zeta_{1}^{-2}}$ \\
\hline$J_{\alpha}$ & $\frac{\left\{c_{\epsilon_{2}} t_{R} \zeta_{1}^{-1}\left[3 \zeta^{-2}+t_{X}\left(1-t_{R}\right)-\sqrt{3} \beta\left(t_{R}+t_{X}-1\right)\right]+s_{\epsilon_{2}} \zeta_{1}^{-2}\left[\sqrt{3} t_{R}^{2}+t_{X} \beta(\sqrt{3} \beta+1)\left(1-t_{R}\right)\right] c_{W}\right.}{6 \zeta^{-1} \zeta_{1}^{-2}}$ \\
\hline$J_{3}$ & $\frac{\left\{c_{\epsilon_{2}} t_{R} t_{X} \zeta_{1}^{-1}\left[2 \sqrt{3} t_{X} \beta+\left(t_{R}-1\right)(\sqrt{3} \beta-1)\right]+s_{\epsilon_{\epsilon}} \zeta_{1}^{-2}\left[-2 \sqrt{3} t_{R}^{2}+t_{X} \beta(\sqrt{3} \beta-1)\left(t_{R}-1\right)\right]\right\} c_{W}}{6 \zeta^{-1} \zeta_{1}^{-2}}$ \\
\hline
\end{tabular}

Table 4. The couplings of $\mathcal{Z}_{R}$ with fermions. 


\begin{tabular}{|c|c|}
\hline$f$ & $g_{V}^{\mathcal{Z}_{\mathcal{R}}^{\prime}}(f)$ \\
\hline$\nu_{a}$ & $\frac{\left\{-s_{\epsilon_{2}} t_{R} \zeta_{1}^{-1}\left[3 \zeta^{-2}-\sqrt{3} \beta t_{X}^{2}+t_{X}\left(1+t_{R}\right)(3+\sqrt{3} \beta)\right]+c_{\epsilon_{2}} \zeta_{1}^{-2}\left[\sqrt{3} t_{R}^{2}+t_{X} \beta(3+\sqrt{3} \beta)\left(1+t_{R}\right)\right]\right\} c_{W}}{6 \zeta^{-1} \zeta_{1}^{-2}}$ \\
\hline$e_{a}$ & $\frac{\left\{s_{\epsilon_{2}} t_{R} \zeta_{1}^{-1}\left[3 \zeta^{-2}+\sqrt{3} \beta t_{X}^{2}-t_{X}\left(1+t_{R}\right)(3+\sqrt{3} \beta)\right]+c_{\epsilon_{2}} \zeta_{1}^{-2}\left[\sqrt{3} t_{R}^{2}+t_{X} \beta(3+\sqrt{3} \beta)\left(1+t_{R}\right)\right]\right\} c_{W}}{6 \zeta^{-1} \zeta_{1}^{-2}}$ \\
\hline$N_{a}$ & $-\frac{\left\{s_{\epsilon_{2}} t_{R} t_{X} \zeta_{1}^{-1}\left[2 \sqrt{3} t_{X} \beta+\left(t_{R}+1\right)(3+\sqrt{3} \beta)\right]+c_{\epsilon_{2}} \zeta_{1}^{-2}\left[2 \sqrt{3} t_{R}^{2}-t_{X} \beta(3+\sqrt{3} \beta)\left(1+t_{R}\right)\right]\right\} c_{W}}{6 \zeta^{-1} \zeta_{1}^{-2}}$ \\
\hline$u_{\alpha}$ & $\frac{\left\{s_{\epsilon_{2}} t_{R} \zeta_{1}^{-1}\left[3 \zeta^{-2}+t_{X}\left(t_{R}+1\right)+\sqrt{3} \beta t_{X}\left(1+t_{R}+t_{X}\right)\right]+c_{\epsilon_{2}} \zeta_{1}^{-2}\left[\sqrt{3} t_{R}^{2}-t_{X} \beta(1+\sqrt{3} \beta)\left(1-t_{R}\right)\right]\right\} c_{W}}{6 \zeta^{-1} \zeta_{1}^{-2}}$ \\
\hline$u_{3}$ & $\frac{\left\{s_{\epsilon_{2}} t_{R} \zeta_{1}^{-1}\left[-3 \zeta^{-2}+t_{X}\left(t_{R}+1\right)+\sqrt{3} t_{X}\left(t_{X}-t_{R}-1\right) \beta\right]+c_{\epsilon_{2}} \zeta_{1}^{-2}\left[\sqrt{3} t_{R}^{2}+t_{X} \beta(\sqrt{3} \beta-1)\left(t_{R}+1\right)\right]\right\} c_{W}}{6 \zeta^{-1} \zeta_{1}^{-2}}$ \\
\hline$d_{\alpha}$ & $\frac{\left\{s_{\epsilon_{2}} t_{R} \zeta_{1}^{-1}\left[-3 \zeta^{-2}+t_{X}\left(t_{R}+1\right)+\sqrt{3} \beta t_{X}\left(t_{X}+t_{R}+1\right)\right]+c_{\epsilon_{2}} \zeta_{1}^{-2}\left[\sqrt{3} t_{R}^{2}-t_{X} \beta(\sqrt{3} \beta+1)\left(t_{R}+1\right)\right]\right\} c_{W}}{6 \zeta^{-1} \zeta_{1}^{-2}}$ \\
\hline$d_{3}$ & $\frac{\left\{s_{\epsilon_{2}} t_{R} \zeta_{1}^{-1}\left[3 \zeta^{-2}+t_{X}\left(t_{R}+1\right)+\sqrt{3} \beta t_{X}\left(t_{X}-t_{R}-1\right)\right]+c_{\epsilon_{2}} \zeta_{1}^{-2}\left[\sqrt{3} t_{R}^{2}+t_{X} \beta(\sqrt{3} \beta-1)\left(t_{R}+1\right)\right]\right\} c_{W}}{6 \zeta^{-1} \zeta_{1}^{-2}}$ \\
\hline$J_{\alpha}$ & $-\frac{\left\{-s_{\epsilon_{2}} t_{R} t_{X} \zeta_{1}^{-1}\left[1+t_{R}+\sqrt{3} \beta\left(1+t_{R}-2 t_{X}\right)\right]+c_{\epsilon_{2}} \zeta_{1}^{-2}\left[2 \sqrt{3} t_{R}^{2}+t_{X} \beta(\sqrt{3} \beta+1)\left(t_{R}+1\right)\right]\right\} c_{W}}{6 \zeta^{-1} \zeta_{1}^{-2}}$ \\
\hline$J_{3}$ & $-\frac{\left\{-s_{\epsilon_{2}} t_{R} t_{X} \zeta_{1}^{-1}\left[1+t_{R}-\sqrt{3} t_{R} \beta-\sqrt{3} \beta\left(1+2 t_{X}\right)\right]+c_{\epsilon_{2}} \zeta_{1}^{-2}\left[2 \sqrt{3} t_{R}^{2}+t_{X} \beta(1-\sqrt{3} \beta)\left(t_{R}+1\right)\right]\right\} c_{W}}{6 \zeta^{-1} \zeta_{1}^{-2}}$ \\
\hline$f$ & $g_{A}^{\mathcal{Z}_{\mathcal{R}}^{\prime}}(f)$ \\
\hline$\nu_{a}$ & $\frac{\left\{s_{\epsilon_{2}} t_{R} \zeta_{1}^{-1}\left[3 \zeta^{-2}-\sqrt{3} \beta t_{X}^{2}+t_{X}\left(t_{R}-1\right)(3+\sqrt{3} \beta)\right]-c_{\epsilon_{2}} \zeta_{1}^{-2}\left[\sqrt{3} t_{R}^{2}+t_{X} \beta(3+\sqrt{3} \beta)\left(t_{R}-1\right)\right]\right\} c_{W}}{6 \zeta^{-1} \zeta_{1}^{-2}}$ \\
\hline$e_{a}$ & $-\frac{\left\{s_{\epsilon_{2}} t_{R} \zeta_{1}^{-1}\left[3 \zeta^{-2}+\sqrt{3} \beta t_{X}^{2}-t_{X}\left(t_{R}-1\right)(3+\sqrt{3} \beta)\right]+c_{\epsilon_{2}} \zeta_{1}^{-2}\left[\sqrt{3} t_{R}^{2}+t_{X} \beta(3+\sqrt{3} \beta)\left(t_{R}-1\right)\right]\right\} c_{W}}{6 \zeta^{-1} \zeta_{1}^{-2}}$ \\
\hline$N_{a}$ & $\frac{\left\{s_{\epsilon_{2}} t_{R} t_{X} \zeta_{1}^{-1}\left[2 \sqrt{3} t_{X} \beta+\left(t_{R}-1\right)(3+\sqrt{3} \beta)\right]+c_{\epsilon_{2}} \zeta_{1}^{-2}\left[2 \sqrt{3} t_{R}^{2}+t_{X} \beta(3+\sqrt{3} \beta)\left(1-t_{R}\right)\right]\right\} c_{W}}{6 \zeta^{-1} \zeta_{1}^{-2}}$ \\
\hline$u_{\alpha}$ & $-\frac{\left\{s_{\epsilon_{2}} t_{R} \zeta_{1}^{-1}\left[3 \zeta^{-2}+t_{X}\left(t_{R}-1\right)+\sqrt{3} \beta t_{X}\left(-1+t_{R}+t_{X}\right)\right]+c_{\epsilon_{2}} \zeta_{1}^{-2}\left[\sqrt{3} t_{R}^{2}+t_{X} \beta(1+\sqrt{3} \beta)\left(1-t_{R}\right)\right]\right\} c_{W}}{6 \zeta^{-1} \zeta_{1}^{-2}}$ \\
\hline$u_{3}$ & $\frac{\left\{s_{\epsilon_{2}} t_{R} \zeta_{1}^{-1}\left[3 \zeta^{-2}+t_{X}\left(t_{R}-1\right)(\sqrt{3} \beta-1)-\sqrt{3} \beta t_{X}^{2}\right]-c_{\epsilon_{2}} \zeta_{1}^{-2}\left[\sqrt{3} t_{R}^{2}+t_{X} \beta(\sqrt{3} \beta-1)\left(t_{R}-1\right)\right]\right\} c_{W}}{6 \zeta^{-1} \zeta_{1}^{-2}}$ \\
\hline$d_{\alpha}$ & $\frac{\left\{s_{\epsilon_{2}} t_{R} \zeta_{1}^{-1}\left[3 \zeta^{-2}+t_{X}\left(1-t_{R}\right)-\sqrt{3} \beta t_{X}\left(t_{X}+t_{R}-1\right)\right]-c_{\epsilon_{2}} \zeta_{1}^{-2}\left[\sqrt{3} t_{R}^{2}+t_{X} \beta(\sqrt{3} \beta+1)\left(1-t_{R}\right)\right] c_{W}\right.}{6 \zeta^{-1} \zeta_{1}^{-2}}$ \\
\hline$d_{3}$ & $-\frac{\left\{s_{\epsilon_{2}} t_{R} \zeta_{1}^{-1}\left[3 \zeta^{-2}+t_{X}\left(t_{R}-1\right)+\sqrt{3} \beta t_{X}\left(t_{X}-t_{R}+1\right)\right]+c_{\epsilon_{2}} \zeta_{1}^{-2}\left[\sqrt{3} t_{R}^{2}+t_{X} \beta(1-\sqrt{3} \beta)\left(1+t_{R}\right)\right]\right\}_{W}}{6 \zeta^{-1} \zeta_{1}^{-2}}$ \\
\hline$J_{\alpha}$ & $\frac{\left\{s_{\epsilon_{2}} t_{R} t_{x} \zeta_{1}^{-1}\left[\left(1-t_{R}\right)+\sqrt{3} \beta\left(1-t_{R}+2 t_{X}\right)\right]+c_{\epsilon_{2}} \zeta_{1}^{-2}\left[2 \sqrt{3} t_{R}^{2}+t_{X} \beta(\sqrt{3} \beta+1)\left(t_{R}-1\right)\right] c_{W}\right.}{6 \zeta^{-1} \zeta_{1}^{-2}}$ \\
\hline$J_{3}$ & $\frac{\left\{s_{\epsilon_{2}} t_{R} t_{X} \zeta_{1}^{-1}\left[2 \sqrt{3} t_{X} \beta+\left(t_{R}-1\right)(\sqrt{3} \beta-1)\right]-c_{\epsilon_{2}} \zeta_{1}^{-2}\left[-2 \sqrt{3} t_{R}^{2}+t_{X} \beta(\sqrt{3} \beta-1)\left(t_{R}-1\right)\right]\right\} c_{W}}{6 \zeta^{-1} \zeta_{1}^{-2}}$ \\
\hline
\end{tabular}

Table 5. The couplings of $\mathcal{Z}_{R}^{\prime}$ with fermions. 
Open Access. This article is distributed under the terms of the Creative Commons Attribution License (CC-BY 4.0), which permits any use, distribution and reproduction in any medium, provided the original author(s) and source are credited.

\section{References}

[1] J. Silk et al., Particle Dark Matter: Observations, Models and Searches, Cambridge University Press, Cambridge (2010).

[2] F.S. Queiroz, W. Rodejohann and C.E. Yaguna, Is the dark matter particle its own antiparticle?, Phys. Rev. D 95 (2017) 095010 [arXiv:1610.06581] [INSPIRE].

[3] R.M. Capdevilla, A. Delgado, A. Martin and N. Raj, Characterizing dark matter at the LHC in Drell-Yan events, Phys. Rev. D 97 (2018) 035016 [arXiv:1709.00439] [INSPIRE].

[4] B.J. Kavanagh, F.S. Queiroz, W. Rodejohann and C.E. Yaguna, Prospects for determining the particle/antiparticle nature of WIMP dark matter with direct detection experiments, JHEP 10 (2017) 059 [arXiv:1706.07819] [INSPIRE].

[5] J.C. Pati and A. Salam, Lepton Number as the Fourth Color, Phys. Rev. D 10 (1974) 275 [Erratum ibid. D 11 (1975) 703] [INSPIRE].

[6] R.N. Mohapatra and J.C. Pati, Left-Right Gauge Symmetry and an Isoconjugate Model of CP-violation, Phys. Rev. D 11 (1975) 566 [InSPIRE].

[7] G. Senjanović and R.N. Mohapatra, Exact Left-Right Symmetry and Spontaneous Violation of Parity, Phys. Rev. D 12 (1975) 1502 [InSPIRE].

[8] P. Minkowski, $\mu \rightarrow$ er at a Rate of One Out of $10^{9}$ Muon Decays?, Phys. Lett. B 67 (1977) 421 [INSPIRE].

[9] G. Senjanović, Spontaneous Breakdown of Parity in a Class of Gauge Theories, Nucl. Phys. B 153 (1979) 334 [INSPIRE].

[10] R.N. Mohapatra and G. Senjanović, Neutrino Mass and Spontaneous Parity Violation, Phys. Rev. Lett. 44 (1980) 912 [INSPIRE].

[11] M. Nemevšek, G. Senjanović and Y. Zhang, Warm Dark Matter in Low Scale Left-Right Theory, JCAP 07 (2012) 006 [arXiv:1205.0844] [INSPIRE].

[12] J. Heeck and S. Patra, Minimal Left-Right Symmetric Dark Matter, Phys. Rev. Lett. 115 (2015) 121804 [arXiv: 1507.01584] [INSPIRE].

[13] C. Garcia-Cely and J. Heeck, Phenomenology of left-right symmetric dark matter, arXiv: 1512.03332 [INSPIRE].

[14] S. Patra and S. Rao, Singlet fermion Dark Matter within Left-Right Model, Phys. Lett. B 759 (2016) 454 [arXiv: 1512.04053] [INSPIRE].

[15] P.S. Bhupal Dev, R.N. Mohapatra and Y. Zhang, Naturally stable right-handed neutrino dark matter, JHEP 11 (2016) 077 [arXiv:1608.06266] [INSPIRE].

[16] M. Singer, J.W.F. Valle and J. Schechter, Canonical Neutral Current Predictions From the Weak Electromagnetic Gauge Group $\mathrm{SU}(3) \times \mathrm{U}(1)$, Phys. Rev. D 22 (1980) 738 [InSPIRE].

[17] J.W.F. Valle and M. Singer, Lepton Number Violation With Quasi Dirac Neutrinos, Phys. Rev. D 28 (1983) 540 [INSPIRE]. 
[18] F. Pisano and V. Pleitez, An $\mathrm{SU}(3) \times \mathrm{U}(1)$ model for electroweak interactions, Phys. Rev. D 46 (1992) 410 [hep-ph/9206242] [INSPIRE].

[19] P.H. Frampton, Chiral dilepton model and the flavor question, Phys. Rev. Lett. 69 (1992) 2889 [INSPIRE].

[20] R. Foot, O.F. Hernandez, F. Pisano and V. Pleitez, Lepton masses in an $\mathrm{SU}(3)_{L} \times \mathrm{U}(1)_{N}$ gauge model, Phys. Rev. D 47 (1993) 4158 [hep-ph/9207264] [INSPIRE].

[21] J.C. Montero, F. Pisano and V. Pleitez, Neutral currents and GIM mechanism in $\mathrm{SU}(3)_{L} \times \mathrm{U}(1)_{N}$ models for electroweak interactions, Phys. Rev. D 47 (1993) 2918 [hep-ph/9212271] [INSPIRE].

[22] R. Foot, H.N. Long and T.A. Tran, $\mathrm{SU}(3)_{L} \otimes \mathrm{U}(1)_{N}$ and $\mathrm{SU}(4)_{L} \otimes \mathrm{U}(1)_{N}$ gauge models with right-handed neutrinos, Phys. Rev. D 50 (1994) R34 [hep-ph/9402243] [INSPIRE].

[23] H.N. Long, The 331 model with right handed neutrinos, Phys. Rev. D 53 (1996) 437 [hep-ph/9504274] [INSPIRE].

[24] M. Reig, J.W.F. Valle and C.A. Vaquera-Araujo, Three-family left-right symmetry with low-scale seesaw mechanism, JHEP 05 (2017) 100 [arXiv:1611.04571] [INSPIRE].

[25] K.S. Babu, X.-G. He and S. Pakvasa, Neutrino Masses and Proton Decay Modes in $\mathrm{SU}(3) \times \mathrm{SU}(3) \times \mathrm{SU}(3)$ Trinification, Phys. Rev. D 33 (1986) 763 [inSPIRE].

[26] H. Nishimura and A. Okunishi, Strong CP problem and nucleon stability in $\mathrm{SU}(3) \times \mathrm{SU}(3) \times \mathrm{SU}(3)$ trinification model, Phys. Lett. B 209 (1988) 307 [INSPIRE].

[27] E.D. Carlson and M.Y. Wang, Trinification and the strong P problem, hep-ph/9211279 [INSPIRE].

[28] S. Willenbrock, Triplicated trinification, Phys. Lett. B 561 (2003) 130 [hep-ph/0302168] [INSPIRE].

[29] J. Sayre, S. Wiesenfeldt and S. Willenbrock, Minimal trinification, Phys. Rev. D 73 (2006) 035013 [hep-ph/0601040] [INSPIRE].

[30] C. Cauet, H. Pas, S. Wiesenfeldt, H. Pas and S. Wiesenfeldt, Trinification, the Hierarchy Problem and Inverse Seesaw Neutrino Masses, Phys. Rev. D 83 (2011) 093008 [arXiv: 1012.4083] [INSPIRE].

[31] A.G. Dias, C.A. de S. Pires and P.S. Rodrigues da Silva, The Left-Right $\mathrm{SU}(3)_{L} \times \mathrm{SU}(3)_{R} \times \mathrm{U}(1)_{X}$ Model with Light, keV and Heavy Neutrinos, Phys. Rev. D 82 (2010) 035013 [arXiv:1003.3260] [INSPIRE].

[32] B. Stech, Trinification Phenomenology and the structure of Higgs Bosons, JHEP 08 (2014) 139 [arXiv: 1403.2714] [INSPIRE].

[33] G.M. Pelaggi, A. Strumia and S. Vignali, Totally asymptotically free trinification, JHEP 08 (2015) 130 [arXiv:1507.06848] [INSPIRE].

[34] C.P. Ferreira, M.M. Guzzo and P.C. de Holanda, Cosmological bounds of sterile neutrinos in $a \mathrm{SU}(3)_{C} \otimes \mathrm{SU}(3)_{L} \otimes \mathrm{SU}(3)_{R} \otimes \mathrm{U}(1)_{N}$ model as dark matter candidates, Braz. J. Phys. 46 (2016) 453 [arXiv: 1509.02977] [INSPIRE].

[35] G.M. Pelaggi, A. Strumia and E. Vigiani, Trinification can explain the di-photon and di-boson LHC anomalies, JHEP 03 (2016) 025 [arXiv: 1512.07225] [INSPIRE]. 
[36] O. Rodríguez, R.H. Benavides, W.A. Ponce and E. Rojas, Flipped versions of the universal 3-3-1 and the left-right symmetric models in $[\mathrm{SU}(3)]^{3}$ : a comprehensive approach, Phys. Rev. D 95 (2017) 014009 [arXiv: 1605.00575] [INSPIRE].

[37] J.E. Camargo-Molina, A.P. Morais, R. Pasechnik and J. Wessén, On a radiative origin of the Standard Model from Trinification, JHEP 09 (2016) 129 [arXiv: 1606.03492] [INSPIRE].

[38] D.T. Huong and P.V. Dong, Left-right asymmetry and $750 \mathrm{GeV}$ diphoton excess, Phys. Rev. D 93 (2016) 095019 [arXiv: 1603.05146] [INSPIRE].

[39] P.V. Dong and D.T. Huong, Left-right model for dark matter, arXiv:1610.02642 [INSPIRE].

[40] M. Reig, J.W.F. Valle and C.A. Vaquera-Araujo, Unifying left-right symmetry and 331 electroweak theories, Phys. Lett. B 766 (2017) 35 [arXiv:1611.02066] [INSPIRE].

[41] C. Hati, S. Patra, M. Reig, J.W.F. Valle and C.A. Vaquera-Araujo, Towards gauge coupling unification in left-right symmetric $\mathrm{SU}(3)_{c} \times \mathrm{SU}(3)_{L} \times \mathrm{SU}(3)_{R} \times \mathrm{U}(1)_{X}$ theories, Phys. Rev. D 96 (2017) 015004 [arXiv: 1703.09647] [INSPIRE].

[42] C. Kownacki, E. Ma, N. Pollard, O. Popov and M. Zakeri, Dark revelations of the $[\mathrm{SU}(3)]^{3}$ and $[\mathrm{SU}(3)]^{4}$ gauge extensions of the standard model, Phys. Lett. B 777 (2018) 121 [arXiv: 1710.00762] [INSPIRE].

[43] J.K. Mizukoshi, C.A. de S. Pires, F.S. Queiroz and P.S. Rodrigues da Silva, WIMPs in a 3-3-1 model with heavy Sterile neutrinos, Phys. Rev. D 83 (2011) 065024 [arXiv:1010.4097] [INSPIRE].

[44] S. Profumo and F.S. Queiroz, Constraining the $Z^{\prime}$ mass in 331 models using direct dark matter detection, Eur. Phys. J. C 74 (2014) 2960 [arXiv:1307.7802] [INSPIRE].

[45] P.V. Dong, H.T. Hung and T.D. Tham, 3-3-1-1 model for dark matter, Phys. Rev. D 87 (2013) 115003 [arXiv: 1305.0369] [inSPIRE].

[46] P.V. Dong, T.P. Nguyen and D.V. Soa, 3-3-1 model with inert scalar triplet, Phys. Rev. D 88 (2013) 095014 [arXiv: 1308.4097] [INSPIRE].

[47] C. Kelso, C.A. de S. Pires, S. Profumo, F.S. Queiroz and P.S. Rodrigues da Silva, A 331 WIMPy Dark Radiation Model, Eur. Phys. J. C 74 (2014) 2797 [arXiv:1308.6630] [INSPIRE].

[48] P.V. Dong, N.T.K. Ngan and D.V. Soa, Simple 3-3-1 model and implication for dark matter, Phys. Rev. D 90 (2014) 075019 [arXiv: 1407.3839] [INSPIRE].

[49] P.V. Dong, C.S. Kim, D.V. Soa and N.T. Thuy, Investigation of Dark Matter in Minimal 3-3-1 Models, Phys. Rev. D 91 (2015) 115019 [arXiv:1501.04385] [INSPIRE].

[50] D. Cogollo, A.X. Gonzalez-Morales, F.S. Queiroz and P.R. Teles, Excluding the Light Dark Matter Window of a 331 Model Using LHC and Direct Dark Matter Detection Data, JCAP 11 (2014) 002 [arXiv: 1402.3271] [INSPIRE].

[51] P.V. Dong, D.T. Huong, F.S. Queiroz and N.T. Thuy, Phenomenology of the 3-3-1-1 model, Phys. Rev. D 90 (2014) 075021 [arXiv: 1405.2591] [INSPIRE].

[52] C. Kelso, H.N. Long, R. Martinez and F.S. Queiroz, Connection of $g-2_{\mu}$, electroweak, dark matter and collider constraints on 331 models, Phys. Rev. D 90 (2014) 113011 [arXiv: 1408.6203] [INSPIRE].

[53] A. Alves, A. Berlin, S. Profumo and F.S. Queiroz, Dark Matter Complementarity and the $Z^{\prime}$ Portal, Phys. Rev. D 92 (2015) 083004 [arXiv:1501.03490] [inSPIRE]. 
[54] Y. Mambrini, S. Profumo and F.S. Queiroz, Dark Matter and Global Symmetries, Phys. Lett. B 760 (2016) 807 [arXiv: 1508.06635] [INSPIRE].

[55] D.T. Huong and P.V. Dong, Neutrino masses and superheavy dark matter in the 3-3-1-1 model, Eur. Phys. J. C 77 (2017) 204 [arXiv:1605.01216] [INSPIRE].

[56] P.V. Dong, Unifying the electroweak and B-L interactions, Phys. Rev. D 92 (2015) 055026 [arXiv: 1505. 06469] [INSPIRE].

[57] D.T. Huong, P.V. Dong, C.S. Kim and N.T. Thuy, Inflation and leptogenesis in the 3-3-1-1 model, Phys. Rev. D 91 (2015) 055023 [arXiv: 1501.00543] [INSPIRE].

[58] Particle Data Group collaboration, C. Patrignani et al., Review of Particle Physics, Chin. Phys. C 40 (2016) 100001 [inSPIRE].

[59] Cherenkov Telescope Array Consortium collaboration, B.S. Acharya et al., Science with the Cherenkov Telescope Array, arXiv:1709.07997 [INSPIRE].

[60] J.D. Ruiz-Alvarez, C.A. de S. Pires, F.S. Queiroz, D. Restrepo and P.S. Rodrigues da Silva, On the Connection of Gamma-Rays, Dark Matter and Higgs Searches at LHC, Phys. Rev. D 86 (2012) 075011 [arXiv:1206.5779] [INSPIRE].

[61] A. Alves, G. Arcadi, P.V. Dong, L. Duarte, F.S. Queiroz and J.W.F. Valle, Matter-parity as a residual gauge symmetry: Probing a theory of cosmological dark matter, Phys. Lett. B 772 (2017) 825 [arXiv : 1612.04383] [INSPIRE].

[62] DAMIC collaboration, A. Aguilar-Arevalo et al., First Direct-Detection Constraints on eV-Scale Hidden-Photon Dark Matter with DAMIC at SNOLAB, Phys. Rev. Lett. 118 (2017) 141803 [arXiv:1611.03066] [INSPIRE].

[63] DAMIC collaboration, A. Aguilar-Arevalo et al., Search for low-mass WIMPs in a $0.6 \mathrm{~kg}$ day exposure of the DAMIC experiment at SNOLAB, Phys. Rev. D 94 (2016) 082006 [arXiv: 1607.07410] [INSPIRE].

[64] O. Civitarese, K.J. Fushimi and M.E. Mosquera, Calculated WIMP signals at the ANDES laboratory: comparison with northern and southern located dark matter detectors, J. Phys. G 43 (2016) 125201 [arXiv:1611.00802] [INSPIRE].

[65] PandaX-II collaboration, C. Fu et al., Spin-Dependent Weakly-Interacting-MassiveParticle-Nucleon Cross Section Limits from First Data of PandaX-II Experiment, Phys. Rev. Lett. 118 (2017) 071301 [arXiv: 1611.06553] [INSPIRE].

[66] XENON100 collaboration, E. Aprile et al., Search for Bosonic Super-WIMP Interactions with the XENON100 Experiment, Phys. Rev. D 96 (2017) 122002 [arXiv:1709.02222] [INSPIRE].

[67] XENON collaboration, E. Aprile et al., First Dark Matter Search Results from the XENON1T Experiment, Phys. Rev. Lett. 119 (2017) 181301 [arXiv:1705.06655] [INSPIRE].

[68] XENON collaboration, E. Aprile et al., Search for Electronic Recoil Event Rate Modulation with 4 Years of XENON100 Data, Phys. Rev. Lett. 118 (2017) 101101 [arXiv:1701.00769] [INSPIRE].

[69] PandaX-II collaboration, X. Cui et al., Dark Matter Results From 54-Ton-Day Exposure of PandaX-II Experiment, Phys. Rev. Lett. 119 (2017) 181302 [arXiv:1708.06917] [inSPIRE].

[70] SuperCDMS collaboration, R. Agnese et al., Results from the Super Cryogenic Dark Matter Search Experiment at Soudan, Phys. Rev. Lett. 120 (2018) 061802 [arXiv:1708.08869] [INSPIRE]. 
[71] A. Alves, S. Profumo and F.S. Queiroz, The dark $Z^{\prime}$ portal: direct, indirect and collider searches, JHEP 04 (2014) 063 [arXiv: 1312.5281] [INSPIRE].

[72] F.S. Queiroz and W. Shepherd, New Physics Contributions to the Muon Anomalous Magnetic Moment: A Numerical Code, Phys. Rev. D 89 (2014) 095024 [arXiv:1403.2309] [InSPIRE].

[73] M. Lindner, M. Platscher and F.S. Queiroz, A Call for New Physics: The Muon Anomalous Magnetic Moment and Lepton Flavor Violation, Phys. Rept. 731 (2018) 1 [arXiv: 1610.06587] [INSPIRE].

[74] XENON collaboration, E. Aprile et al., Physics reach of the XENON1T dark matter experiment, JCAP 04 (2016) 027 [arXiv: 1512.07501] [INSPIRE].

[75] LUX, LZ collaborations, M. Szydagis, The Present and Future of Searching for Dark Matter with $L U X$ and $L Z$, PoS (ICHEP2016) 220 [arXiv: 1611.05525] [INSPIRE]. 